\title{
Analytically-Supported Hybrid Photonic-Plasmonic Crystal Design Using Artificial Neural Networks
}

Jorge-Alberto Peralta-Ángeles ( $\nabla_{\text {jperalta@ciencias.unam.mx })}$

Universidad Nacional Autónoma de México https://orcid.org/0000-0001-5577-9718

Jorge-Alejandro Reyes-Esqueda

Universidad Nacional Autónoma de México

\section{Research Article}

Keywords: Nanophotonics, Plane-wave expansion, Artificial neural networks, Hybrid photonic-plasmonic crystals

Posted Date: January 3rd, 2022

DOI: https://doi.org/10.21203/rs.3.rs-1154455/v1

License: (c) (1) This work is licensed under a Creative Commons Attribution 4.0 International License. Read Full License 


\title{
Analytically-supported hybrid photonic-plasmonic crystal design using Artificial Neural Networks
}

\author{
Jorge-Alberto Peralta-Ángeles ${ }^{1 * \dagger}$ and Jorge-Alejandro Reyes-Esqueda ${ }^{1^{* \dagger}}$ \\ ${ }^{1}$ Instituto de Física, Universidad Nacional Autónoma de México, Circuito de la \\ Investigación Científica, Ciudad Universitaria, Coyoacán, 04510, Ciudad de México, \\ México. \\ *Corresponding authors. E-mails: jperalta@ ciencias.unam.mx; reyes@ fisica.unam.mx; \\ $\uparrow$ These authors contributed equally to this work.
}

\begin{abstract}
An analytical and numerical study of hybrid photonic-plasmonic crystals is presented. The proposed theoretical model describes a system composed of a dielectric photonic crystal on a metallic thin film. To show the validity and usefulness of the model, four particular structures are analyzed, a one-dimensional crystal and three lattices of two-dimensional crystals. The model can calculate the photonic band structure of photonic-plasmonic crystals as a function of structural characteristics, showing two partial bandgaps for a square lattice, and complete bandgaps for triangular lattices. Furthermore, using a particular high-symmetry path, a full bandgap emerges in rectangular lattices, even with a small index of refraction contrast. Using the analytical model, a dataset is generated to train an artificial neural network to predict the center and width of the bandgap, that is, the forward design. In addition, an artificial neural network is trained to tune the optical response, that is, to perform the inverse design. The analytical results are consistent with the physics of the system studied and are supported by numerical simulations. Moreover, the prediction accuracy of the artificial neural networks is better than 95\%. Overall, this paper reports a useful tool for tuning the optical properties of hybrid photonic-plasmonic crystals with potential applications in waveguides, nanocavities, mirrors, etc.
\end{abstract}

Keywords: Nanophotonics, Plane-wave expansion, Artificial neural networks, Hybrid photonic-plasmonic crystals

\section{Introduction}

Photonic crystals are media whose index of refraction is periodically modulated in space. This variation in the index of refraction produces bandgaps in the photonic band structure, which physically results in the inhibition of light propagation in the medium for some frequencies ranges. Such an optical effect can be applied in various photonic devices, including filters, reflectors, and waveguides [1-3].

On the other hand, surface plasmon polaritons (SPPs) are electromagnetic excitations arising at a dielectric-metal interface. The optical properties of these electromagnetic excitations are of great interest, especially in technologies involving photonic devices [4].

As for waveguides based on photonic crystals, it is possible to use them in plasmonic devices. There is experimental evidence of an analogous photonic bandgap effect in periodic arrays of gold nanoparticles [5]. In addition, there are experimental observations of waveguiding of SPPs through bent linear defects fabricated in periodic arrays of gold 
nanoparticles [6-8]. Similarly, these periodic arrays can be used as mirrors to modify the propagation of SPPs [9].

Furthermore, experimental studies have analyzed the influence of different characteristics of plasmonic crystals in the photonic bandgap. Such characteristics are the lattice period or lattice constant, filling fraction, and crystal orientation effect $[10,11]$. These experimental results suggest that the propagation of SPPs can be controlled through the appropriate characteristics of the plasmonic crystal.

Metallic photonic crystals have proven to be efficient in producing bandgaps, however, this effect can also be achieved with dielectric photonic crystals. Numerical and experimental studies have shown the possibility of using dielectric ridge stacks as onedimensional plasmonic crystals to open bandgaps in the SPP dispersion relation [12]. The results of this last work suggest the application of these structures as a mechanism to control SPP propagation.

In two-dimensional arrays, dielectric photonic crystals have been numerically analyzed to implement them as cavities [13, 14]. The results obtained from numerical studies show that these hybrid structures, photonic-plasmonic crystals, are capable of opening bandgaps in the dispersion ratio. This suggests that they can be applied in a wide range of plasmonic devices such as nanocavities, nanolasers, reflectors or waveguides [13, $14]$.

On the other hand, several theoretical analyses have been developed for modeling the optical response of these plasmonic systems. Some of these studies are based on the multiple-scattering dipole approach [15], on the Lippmann-Schwinger integral equation method [16], and on the homogeneous form of the reduced Rayleigh equation [17].

As for conventional photonic crystals, one way to know their optical properties is to calculate their photonic band structure. There are several methods to do so, where one of the most widely used is the plane-wave expansion method (PWEM). Although this method is effective for calculating the band structure of dielectric photonic crystals, it also works for calculating the band structure of frequency-dependent media. For example, the PWEM has been applied to calculate the band structure of a silicon photonic crystal over an aluminum film [18].

Another way to calculate the photonic band structure of these optical devices is based on perturbation theory. From the band structure of a dielectric photonic crystal, perturbation theory is applied to analyze the effect of a frequency-dependent dielectric function [19]. However, this method is effective when the variation of the dielectric function is less than $1 \%[3]$.

In an opposite direction, the inverse-design consists in the retrieval of the suitable structure for a particular desired optical response [20]. However, it sometimes requires a search for different system characteristics, starting from a random design, and comparing the result with the target response [21]. This search, called optimization, is performed iteratively and need being carried out too many times to achieve the desired result [21].

In contrast to the above, machine learning algorithms, such as artificial neural networks (ANNs), are efficient tools to design photonic structures [20, 21]. ANNs can be applied in two different ways. The first is to use the structural parameters to predict the optical response, which is useful to avoid the optimization loop, which can sometimes be computationally expensive. This process is known as forward-design [21]. The second approach to the ANNs is using the inverse-design mentioned before. The aim is to predict the structural properties of the photonic system from the desired optical response [20, 21]. 
In this work, an analytical model based on PWEM is proposed to calculate the photonic band structure of hybrid photonic-plasmonic crystals ( $\mathrm{PhPl}$ crystals). These hybrid structures are composed of a dielectric photonic crystal, whose dielectric function is not frequency-dependent, supported on a thin film of gold and silver. Since the dielectric functions of gold and silver are frequency-dependent, a nonlinear eigenvalue equation is needed to solve the corresponding Helmholtz equation.

From the general model, the photonic band structure of four particular systems is studied to test the proposed model and show its usefulness. The analyzed photonic devices are experimentally feasible, since they are composed of a dielectric photonic crystal formed by a periodic array of PMMA structures supported on a thin metallic film.

Using the analytical model, a dataset was constructed to train an ANN. This ANN is used to predict the optical response of a hybrid $\mathrm{PhPl}$ crystal without the need to run a computationally expensive code (forward-design). Furthermore, this ANN is useful to build a dataset larger than the first one to train a second ANN.

With the last data set, this second ANN was trained to predict the structural parameters for a given specific optical response (inverse-design), that is, this ANN is a useful tool to tune the optical response. To validate the results obtained from this ANN, the predicted value is compared to the results obtained with the analytical model, and both are supported by numerical simulations.

\section{Analytical model}

The PWEM is a widely used tool to solve the wave equation for photonic crystals. In this method, both the electric field and the inverse of dielectric functions are expressed as Fourier series:

$$
\begin{gathered}
\mathbf{E}(\mathbf{r})=\sum_{\mathbf{G}} \mathcal{E}_{\mathbf{k} n}(\mathbf{G}) e^{i(\mathbf{k}+\mathbf{G}) \cdot \mathbf{r}}, \\
\frac{1}{\epsilon(\mathbf{G})}=\sum_{\mathbf{G}} e^{i \mathbf{G} \cdot \mathbf{r}}
\end{gathered}
$$

where $\mathcal{E}_{\mathbf{k} n}(\mathbf{G})$ and $\xi(\mathbf{G})$ are the Fourier coefficients of the expansion [1-3].

Introducing both equations in the Helmholtz's equation, one obtains the master equation for photonic crystals for a TM polarized electromagnetic wave

$$
-\sum_{\mathbf{G} \prime} \xi\left(\mathbf{G}-\mathbf{G}^{\prime}\right)\left(\mathbf{k}+\mathbf{G}^{\prime}\right) \times\left\{\left(\mathbf{k}+\mathbf{G}^{\prime}\right) \times \mathcal{E}_{\mathbf{k} n}\left(\mathbf{G}^{\prime}\right)\right\}=\frac{\omega_{\mathbf{k n}}^{2}}{c^{2}} \varepsilon_{\mathbf{k} n}(\mathbf{G}),
$$

where $\mathbf{G}$ and $\mathbf{G}^{\prime}$ are the reciprocal vectors, $\mathbf{k}$ is the wave vector, and $\omega_{\mathbf{k} n}$ are the eigenfrequencies $[1,2]$.

This equation can be expressed as a matrix equation as follows

$$
\widehat{\Xi}^{\prime} \widehat{\mathbf{K}} \mathcal{E}=\widehat{\mathbf{M}} \mathcal{E}=\frac{\omega^{2}}{c^{2}} \mathcal{E},
$$


where $\widehat{\mathbf{\Xi}}^{\prime}$ is a matrix constructed with the coefficients $\xi, \widehat{\mathbf{K}}$ is a matrix formed by the vectors $\mathbf{k}$ and $\mathbf{G}$, and $\mathcal{E}$ is a vector constructed with the coefficients $\mathcal{E}_{\mathbf{k} n}$. The last equation is an eigenvalue equation and can be considered as solved when the eigenvalues of the matrix $\widehat{\mathbf{M}}$ are found [1-3].

When the dielectric function is non-dependent on the frequency, the eigenfrequencies are obtained by solving equation (4). However, if the dielectric function is frequencydependent, as it is the case for SPPs, solving the equation is not straightforward since it is a nonlinear eigenvalue equation.

The analytical model is based on the PWEM, however, the problem to be solved is a nonlinear eigenvalue equation. The key to solving this equation is to find how the inverse of the dielectric function depends on the frequency over a given range of wavelengths. The above allows to convert the nonlinear eigenvalue problem into a linear eigenvalue problem to solve equation (4).

Let be a dielectric-metal interface that gives rise to a typical SPP propagation. The effective index of refraction is defined as [4]

$$
n_{e f f}=\sqrt{\frac{\epsilon_{d} \epsilon_{m}}{\epsilon_{d}+\epsilon_{m}}},
$$

so that the inverse of the dielectric function is

$$
\frac{1}{\epsilon_{e f f}}=\frac{\epsilon_{d}+\epsilon_{m}}{\epsilon_{d} \epsilon_{m}},
$$

where $\epsilon_{d}$ and $\epsilon_{m}$ are the dielectric functions of dielectric and metallic media, respectively.

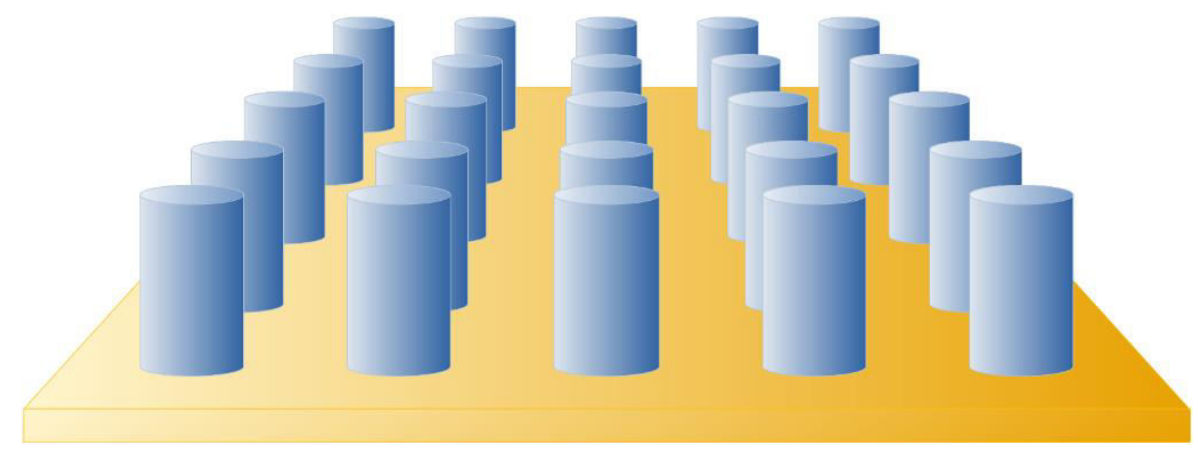

Figure 1. Physical system composed of a dielectric photonic crystal on a gold or silver thin

The system under analysis is a photonic crystal made up of two dielectrics, one with permittivity $\epsilon_{a}$ and the other with permittivity $\epsilon_{b}$. Both dielectric functions are considered to be non-dependent of the frequency. This photonic crystal is supported on a metallic thin film as shown in Fig. 1, so there are two interfaces in a unit cell. In this work, the base is the repeating element, that is, the interface formed by the dielectric $b$ and the metallic thin film. 
Figure 2 shows a unit cell of a rectangular lattice with three different bases for one of the dielectrics: an arbitrary shape, a rectangular shape, and a circular shape base.

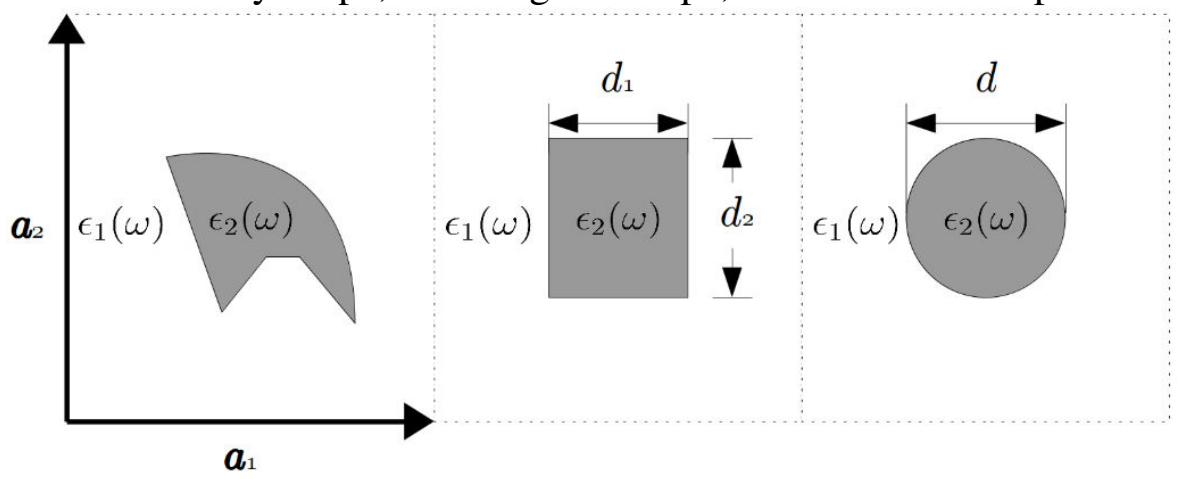

Figure 2. Unit cell of a rectangular lattice with several shapes for one of the dielectrics.

For the interface represented by the white zone in the unit cell, the effective dielectric function is described by $\epsilon_{1}=\epsilon_{a} \epsilon_{m} /\left(\epsilon_{a}+\epsilon_{m}\right)$, while for the interface represented by the gray zone, the effective dielectric function is described by $\epsilon_{1}=\epsilon_{b} \epsilon_{m} /\left(\epsilon_{b}+\epsilon_{m}\right)$. In general, the inverse of the effective dielectric function into all of the unit cell is given by

$$
\frac{1}{\epsilon_{e f f}}=\frac{1}{\epsilon_{1}(\omega)}+\left[\frac{1}{\epsilon_{2}(\omega)}-\frac{1}{\epsilon_{1}(\omega)}\right] F(\mathbf{r})=\frac{1}{\epsilon_{1}(\omega)}+\Delta \xi F(\mathbf{r}),
$$

with $F(\mathbf{r})=1$, if $\mathbf{r}$ is within the gray zone, and $F(\mathbf{r})$ otherwise. It is important to note that the quantity $\Delta \xi$ in the second term does not depend on the frequency.

This way, the Fourier coefficients $\xi$ are given by

$$
\xi(\mathbf{G})=\frac{1}{\epsilon_{1}(\omega)} \delta^{2}(\mathbf{G})+\frac{1}{\mathrm{~A}_{0}} \Delta \xi \mathcal{F}(\mathbf{G}),
$$

where $A_{0}$ is the unit cell area, and $\mathcal{F}(\mathbf{G})$ is the Fourier transform of $F(\mathbf{r})$. The coefficient depends on the geometry of the base and the symmetry of the crystal lattice, since $\mathbf{G}$ is defined by the crystal lattice vectors.

Thus, the matrix $\widehat{\boldsymbol{\Xi}}^{\prime}$ in equation (4) has two terms, one frequency-dependent and one frequency-independent, so that it can be written as

$$
\widehat{\Xi}^{\prime}=\widehat{\Xi}_{0}(\boldsymbol{\omega})+\widehat{\Xi},
$$

where $\widehat{\Xi}$ is constructed by terms of $\frac{1}{A_{0}} \Delta \xi \mathcal{F}(\mathbf{G})$ and $\widehat{\Xi}_{0}$ is given by

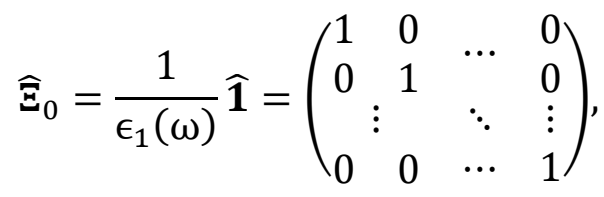

Considering this, equation (4) becomes 


$$
\left(\frac{1}{\epsilon(\omega)} \widehat{\mathbf{1}}+\widehat{\Xi}\right) \widehat{\mathbf{K}} \boldsymbol{\varepsilon}=\frac{\omega^{2}}{c^{2}} \mathcal{\varepsilon},
$$

The inverse of the dielectric function described by equation (6) for dielectric-gold or dielectric-silver interfaces can be approximated, over a suitable frequency range, by a function given as follows

$$
\frac{1}{\epsilon_{e f f}(\omega)}=\frac{1}{\epsilon_{d}}+\gamma_{1}\left(\frac{\omega}{c}-b\right)^{-2}+\gamma_{2}\left(\frac{\omega}{c}-b\right)^{-1}+\gamma_{3}\left(\frac{\omega}{c}-b\right)^{-2},
$$

where parameters $\gamma_{i}$ and $b$ are determined as a function of the dielectric constants $\epsilon_{d}$ and $\epsilon_{m}$.

With the change of variable $\alpha=\omega / c-b$, the inverse of the dielectric functions $1 / \epsilon_{1}(\omega)$ and $1 / \epsilon_{2}(\omega)$ are

$$
\begin{aligned}
& \frac{1}{\epsilon_{1}}=\frac{1}{\epsilon_{a}}+\gamma_{1} \alpha^{-2}+\gamma_{2} \alpha^{-1}+\gamma_{3} \alpha, \\
& \frac{1}{\epsilon_{2}}=\frac{1}{\epsilon_{b}}+\gamma_{1} \alpha^{-2}+\gamma_{2} \alpha^{-1}+\gamma_{3} \alpha,
\end{aligned}
$$

As an example, Fig. 3 shows the inverse of dielectric function data for an air-gold interface (black dots), and for a PMMA-gold interface (gray dots), in the range of wavelengths 548.6-1937 $\mathrm{nm}$. In both cases, the inverse of the dielectric function was calculated using Johnson \& Christy data of permittivity for gold [22]. The continuous lines are the data fitted to the equation (12).

This way, equation (11) 
Wavelenght $(\lambda)[\mu \mathrm{m}]$

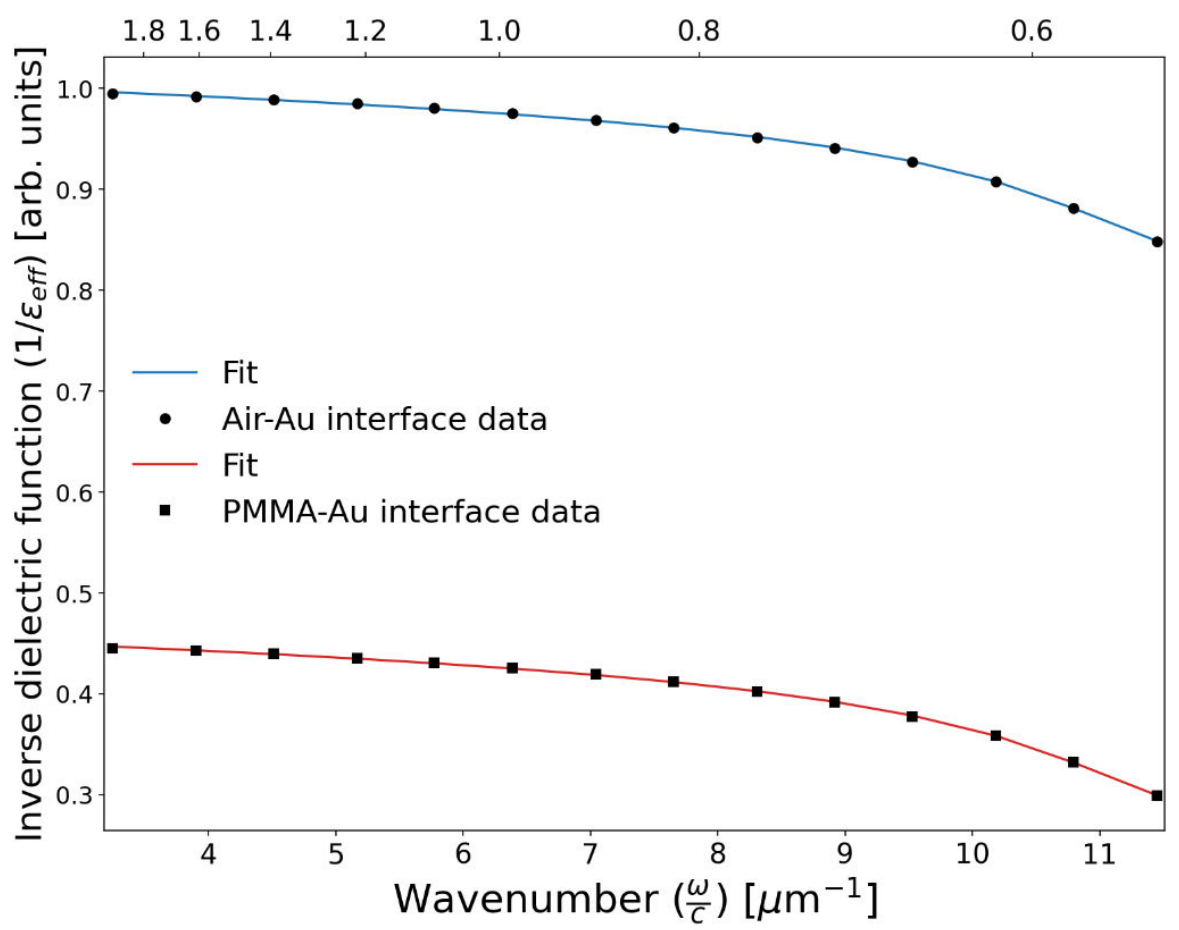

Figure 3. Inverse of dielectric function for two different dielectricgold interfaces.

This way, equation (11) is expressed by

$$
\left(\gamma_{1} \alpha^{-2} \widehat{\mathbf{1}}+\gamma_{2} \alpha^{-1} \widehat{\mathbf{1}}+\frac{1}{\epsilon_{a}} \widehat{\mathbf{1}}+\widehat{\Xi}+\gamma_{3} \alpha \widehat{\mathbf{1}}\right) \widehat{\mathbf{K}} \boldsymbol{E}=\left(b^{2}+2 b \alpha+\alpha^{2}\right) \mathcal{E},
$$

ordering the terms

$$
\left[\alpha^{-2} \gamma_{1} \widehat{\mathbf{K}}+\alpha^{-1} \gamma_{2} \widehat{\mathbf{K}}+\left(\frac{1}{\epsilon_{a}} \widehat{\mathbf{K}}+\widehat{\Xi} \widehat{\mathbf{K}}-b^{2} \widehat{\mathbf{1}}\right)+\alpha\left(\gamma_{3} \widehat{\mathbf{K}}-2 b \widehat{\mathbf{1}}\right)\right] \mathcal{E}=\alpha^{2} \mathcal{E} .
$$

With $\widehat{\mathbf{A}}=\gamma_{1} \widehat{\mathbf{K}}, \widehat{\mathbf{B}}=\gamma_{2} \widehat{\mathbf{K}}, \widehat{\mathbf{C}}=\left(\frac{1}{\epsilon_{a}} \widehat{\mathbf{1}}+\widehat{\Xi}\right) \widehat{\mathbf{K}}-b^{2} \widehat{\mathbf{1}}$ and $\widehat{\mathbf{D}}=\gamma_{3} \widehat{\mathbf{K}}-2 b \widehat{\mathbf{1}}$, equation (16) is rewritten as

$$
\left(\alpha^{-2} \widehat{\mathbf{A}}+\alpha^{-1} \widehat{\mathbf{B}}+\widehat{\mathbf{C}}+\alpha \widehat{\mathbf{D}}\right) \mathcal{E}=\alpha^{2} \mathcal{E} .
$$

Now, the problem has become a nonlinear eigenvalue equation. However, it can be solved with an extended matrix $\widehat{\mathbf{M}}_{\text {ext }}$ to treat it as a linear eigenvalue problem [23, 24]. This extended matrix acts over an extended vector $\mathcal{E}_{\text {ext }}$ as follows

$$
\widehat{\mathbf{M}}_{\text {ext }} \mathcal{E}_{\text {ext }}=\left(\begin{array}{cccc}
\widehat{\mathbf{0}} & \widehat{\mathbf{1}} & \widehat{\mathbf{0}} & \widehat{\mathbf{0}} \\
\widehat{\mathbf{0}} & \widehat{\widehat{\mathbf{0}}} & \widehat{\mathbf{1}} & \widehat{\mathbf{0}} \\
\widehat{\mathbf{0}} & \widehat{\mathbf{0}} & \widehat{\mathbf{0}} & \widehat{\mathbf{1}} \\
\widehat{\mathbf{A}} & \widehat{\mathbf{B}} & \widehat{\mathbf{C}} & \widehat{\mathbf{D}}
\end{array}\right)\left(\begin{array}{c}
\alpha^{-2} \mathcal{E} \\
\alpha^{-1} \mathcal{E} \\
\mathcal{E} \\
\alpha \mathcal{E}
\end{array}\right)=\left(\begin{array}{c}
\alpha^{-1} \mathcal{E} \\
\mathcal{E} \\
\alpha \mathcal{E} \\
\left(\alpha^{-2} \widehat{\mathbf{A}}+\alpha^{-1} \widehat{\mathbf{B}}+\widehat{\mathbf{C}}+\alpha \widehat{\mathbf{D}}\right) \mathcal{E}
\end{array}\right)=
$$




$$
\left(\begin{array}{c}
\alpha^{-1} \mathcal{E} \\
\mathcal{E} \\
\alpha \mathcal{E} \\
\left(\alpha^{-2} \widehat{\mathbf{A}}+\alpha^{-1} \widehat{\mathbf{B}}+\widehat{\mathbf{C}}+\alpha \widehat{\mathbf{D}}\right) \mathcal{E}
\end{array}\right)=\alpha\left(\begin{array}{c}
\alpha^{-2} \mathcal{E} \\
\alpha^{-1} \mathcal{E} \\
\mathcal{E} \\
\alpha \mathcal{E}
\end{array}\right),
$$

To obtain the eigenfrequencies $\omega$, it is sufficient to find the eigenvalues of the matrix $\widehat{\mathbf{M}}_{\text {ext }}$, simplifying enormously the mathematical problem.

\subsection{Particular structures}

The proposed photonic crystal consists of PMMA pillars of either rectangular or elliptical cross-sections immersed in air. It is worth mentioning that square and circular crosssections are considered particular cases of rectangular and elliptical cross-sections. The index of refraction of PMMA has small variations in the 550-2000 nm range and does not represent a significant change in the PMMA-gold dispersion relation if its dielectric function is assumed to be constant. Figure 4 shows the SPP dispersion relation for a PMMA-gold interface.

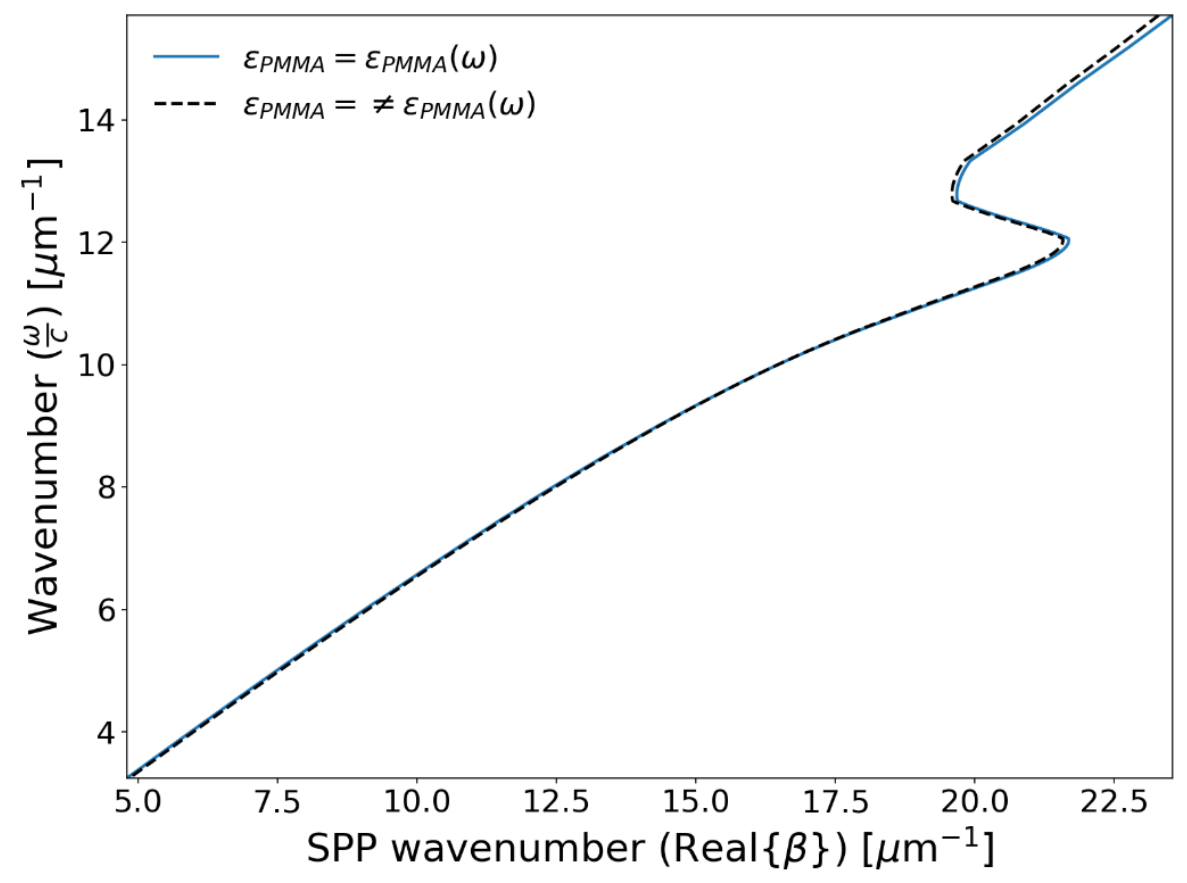

Figure 4. SPP dispersion relation for a PMMA-gold interface.

The dashed line is the dispersion relation considering a frequency-dependent PMMA dielectric function, as in reference [25]. The solid gray line is the dispersion relation considering a constant PMMA dielectric function. The values of the dielectric functions are $\epsilon_{P M M A}=\epsilon_{b}=2.2$ for PMMA, taking the value of PMMA dielectric function at $\lambda=633$ $\mathrm{nm}$ [25]; and $\epsilon_{\text {air }}=\epsilon_{a}=1$ for air, such that the dielectric constants contrast is $\epsilon_{a} / \epsilon_{b}=$ 2.2 .

As an example, Fig. 5 shows the inverse of dielectric function data for an air-gold interface (black circles), and for a PMMA-gold interface (gray circles), in the wavelength range 548.6-1937 nm. The black and gray squares are the inverse of dielectric function data 
for an air-silver and a PMMA-silver interfaces in the range of wavelengths from 331.50 to $1937 \mathrm{~nm}$, respectively. For all the interfaces, the inverse of the dielectric function is calculated using Johnson \& Christy data of permittivity for gold and silver [22]. The solid and dashed lines are the inverse of dielectric function data fitted to equation (12).

Wavelenght $(\lambda)[\mu \mathrm{m}]$

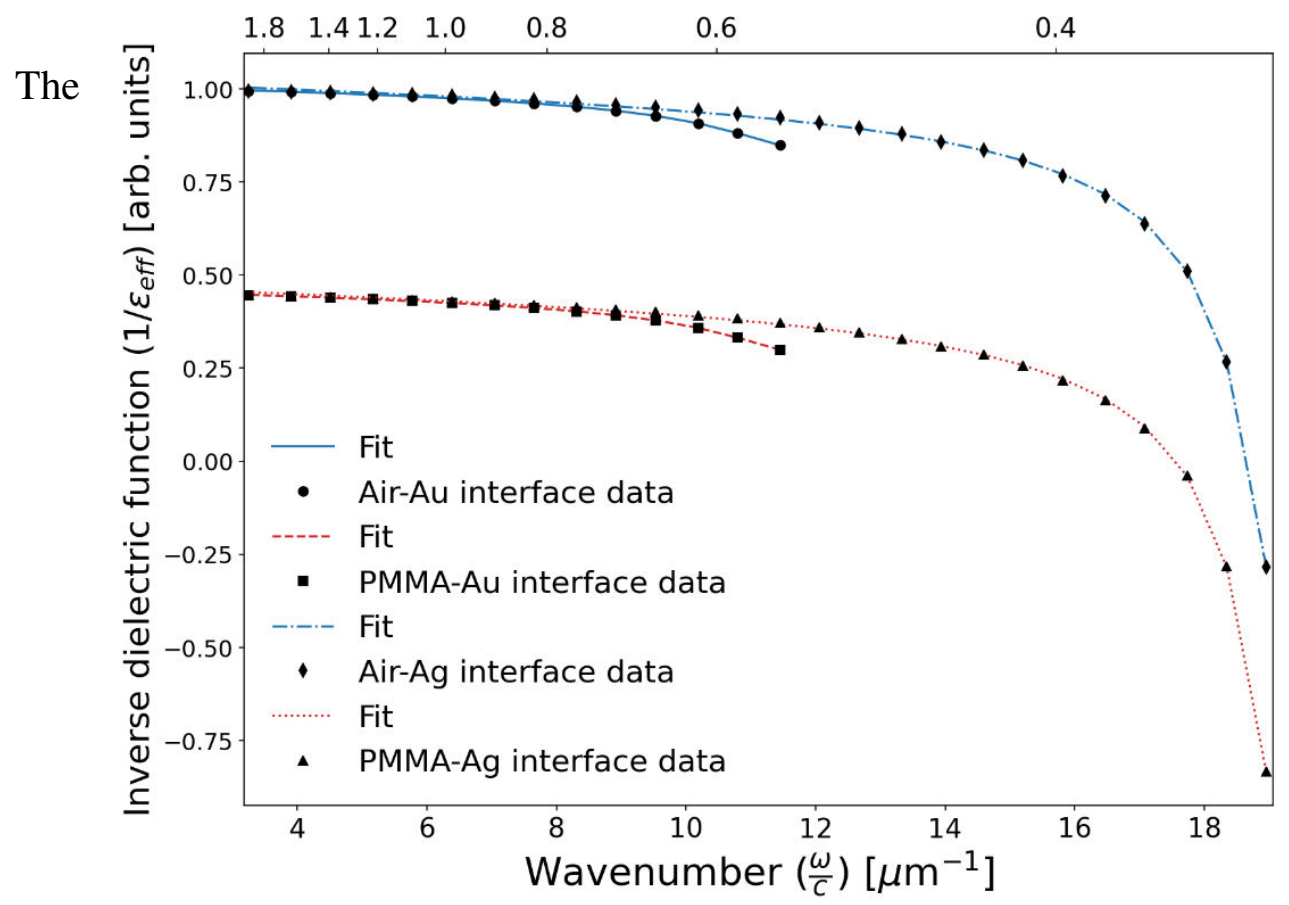

Figure 5. Inverse of dielectric function for four different dielectricgold and dielectric-silver

wavelength ranges for data fitting were chosen to avoid the maximum value of the effective index of refraction, since, near that value, SPP propagation is considerably attenuated. The maximum value of the effective index of refraction for a dielectric-gold interface is located at approximately $520 \mathrm{~nm}$, while for a dielectric-silver interface it occurs approximately at $340 \mathrm{~nm}$.

The data fit parameters for the dielectric-gold interface are $\gamma_{1}=0.115218, \gamma_{2}=$ $0.266632, \gamma_{3}=-0.002649$ and $b=12.368228$. For the dielectric-silver interface, the data fit parameters are $\gamma_{1}=0.153906, \gamma_{2}=0.901489, \gamma_{3}=-0.003654$ and $b=$ 19.361642 .

In the case of a dielectric-gold interface, the fit of the data of the inverse of the effective index of refraction has an asymptotic behavior at $b=12.368228$, which is equivalent to a wavelength of approximately $508 \mathrm{~nm}$. For a dielectric-silver interface, the asymptotic behavior is found at $b=19.361642$, corresponding to a wavelength of approximately $324 \mathrm{~nm}$.

To solve equation (18) for the four chosen different crystal lattices as a function of the lattice length and base size for two bases with different geometries, a python code was written. 


\section{Machine learning algorithms}

To tune the optical response of the photonic crystals, several machine learning algorithms were trained. However, the best results in predicting the physical characteristics of the photonic crystal were obtained using polynomial regression for the square lattice, and an artificial neural network (ANN) for triangular and rectangular lattices.

Table 1. Parameters to generate data for the three lattices and two bases.

\begin{tabular}{|c|c|c|c|c|}
\hline \multirow{2}{*}{\multicolumn{2}{|c|}{ Crystal parameters }} & \multicolumn{3}{|c|}{ Lattice } \\
\hline & & Square & Triangular & Rectangular \\
\hline \multicolumn{2}{|c|}{$a[\mathrm{~nm}]$} & $200-700$ & $300-700$ & - \\
\hline \multicolumn{2}{|c|}{$a_{x}[\mathrm{~nm}]$} & - & - & $0-1$ \\
\hline \multicolumn{2}{|c|}{$a_{y}$} & - & - & $1.2 a_{x}-1.8 a_{y}$ \\
\hline \multirow{2}{*}{$f_{x}$} & Rectangular & $0-1$ & $0-(3-\sqrt{3})$ & $0-1$ \\
\hline & Elliptical & $0-1$ & $0-1$ & $0-1$ \\
\hline \multirow{2}{*}{$f_{y}$} & Rectangular & $0-1$ & $0-(3-\sqrt{3})$ & $0-1$ \\
\hline & Elliptical & $0-1$ & $0-1$ & $0-1$ \\
\hline
\end{tabular}

The data sets necessary to train the algorithms were generated using the analytical model. To calculate the photonic band structure as a function of the lattice and the base sizes, these quantities were varied as shown in table 1 . With the band structure information, the gap width $\Delta \lambda$, and the gap center $\lambda_{c}$, were calculated for all the parameters. 
The filling fractions $f_{x}$ and $f_{y}$, in $x$ and $y$ directions, respectively, are defined as $f_{x}=d_{x} / a$ and $f_{y}=d_{y} / a$, for the square and triangular lattices, and $f_{x}=d_{x} / a_{x}$ and $f_{y}=d_{y} / a_{y}$ for the rectangular lattice. The parameters $d_{x}$ and $d_{y}$ are the characteristic

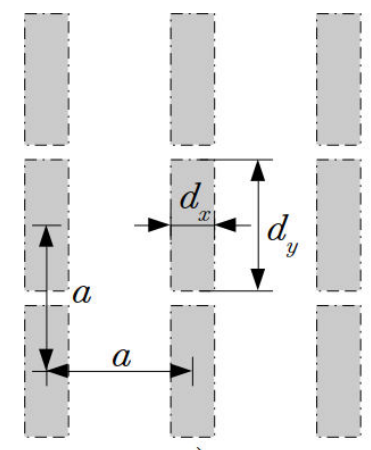

a)

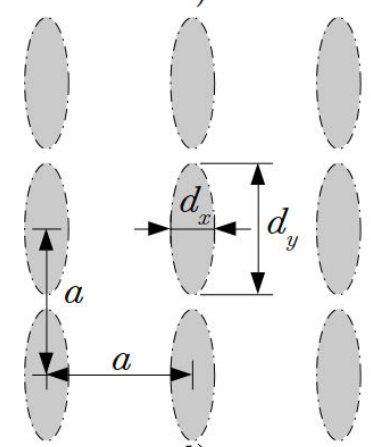

d)

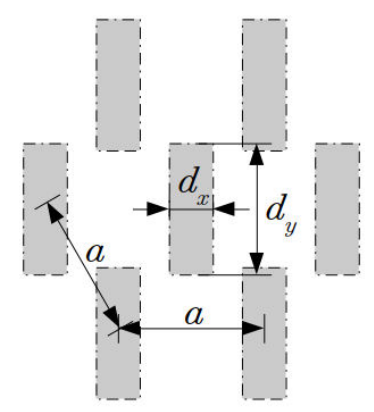

b)

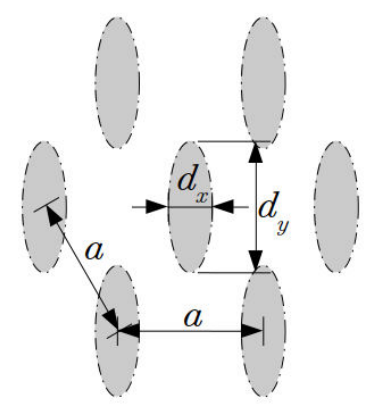

e)

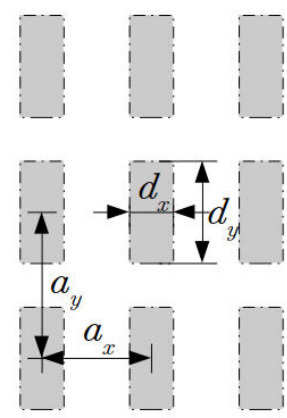

c)

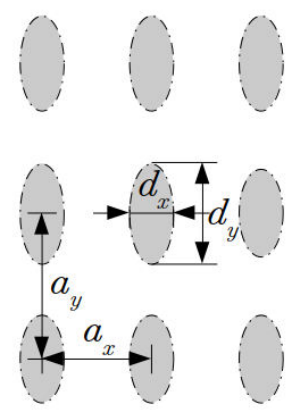

f)

Figure 6. Three two-dimensional lattices with rectangular and elliptical bases.

sizes of the base. For a rectangular base, they are the lengths of the sides in $x$ and $y$ directions, and for an elliptical base, they are the lengths of the axes of the ellipse in $x$ and $y$ directions, respectively, as shown in Fig. 6. As mentioned above, the square and circular bases are particular cases of the rectangular and elliptical bases, when $d_{x}=d_{y}=d$.

For the square lattice, the lattice size is varied in steps of $50 \mathrm{~nm}$. The step of the filling fraction variation was 0.05 for the elliptical base, and 0.1 for the rectangular base.

On the other hand, for the triangular lattice, the variation of the lattice size is the same as the square lattice, $50 \mathrm{~nm}$. The variation of the filling fraction for the elliptical base was 0.043 . For a rectangular base, the step of variation was 0.036 .

In the case of the rectangular lattice, the lattice size in $x$ direction is varied in steps of $50 \mathrm{~nm}$, while the size in $y$ direction is varied in steps of 0.1 , from $1.2 a_{x}$ to $1.8 a_{x}$. The step of the filling fraction for both, the elliptical and the rectangular bases, was 0.05 .

To train the algorithms, polynomial regression for the square lattice was tested using various degrees. For a polynomial of degree 2, the prediction accuracy is better than $90 \%$. However, to calculate the optical response, a polynomial of degree 6 was used for training.

On the other hand, for triangular and rectangular lattices, an ANN was trained to predict the optical response $\left(\Delta \lambda\right.$ and $\left.\lambda_{c}\right)$ as a function of the lattice size $(a)$, and base size 
$\left(d_{x}\right.$ and $\left.d_{y}\right)$. This ANN architecture is shown in Fig. 7. This ANN has five hidden layers, where the first and fifth layers have 100 neurons, the second and fourth layers have 200

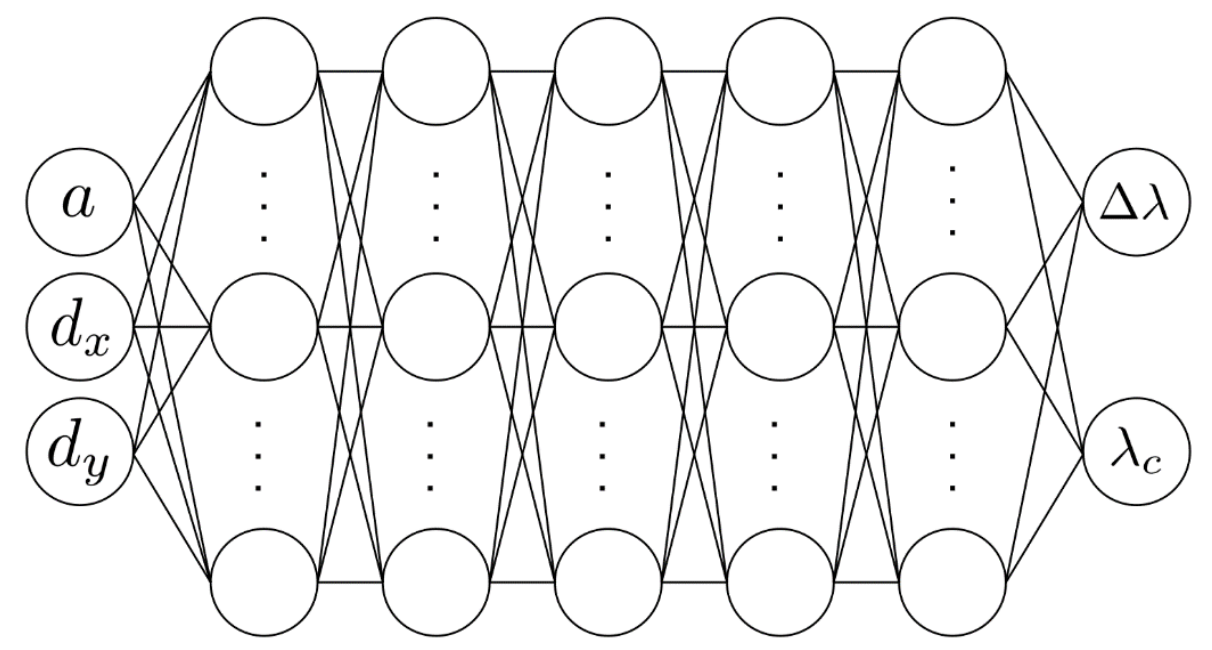

Figure 7. ANN architecture to predict the optical response of triangular lattice.

neurons, and the third layer has 300 neurons.

The ANN was trained with 12486 samples, $70 \%$ of which were used to training, $15 \%$ to testing, and $15 \%$ was used for cross-validation. The optimizer used was Adam, with a learning rate of 0.004 , the loss function used was mean square error, and the metric used was accuracy. In addition, a batch size of 6000 and 200 epochs were used.

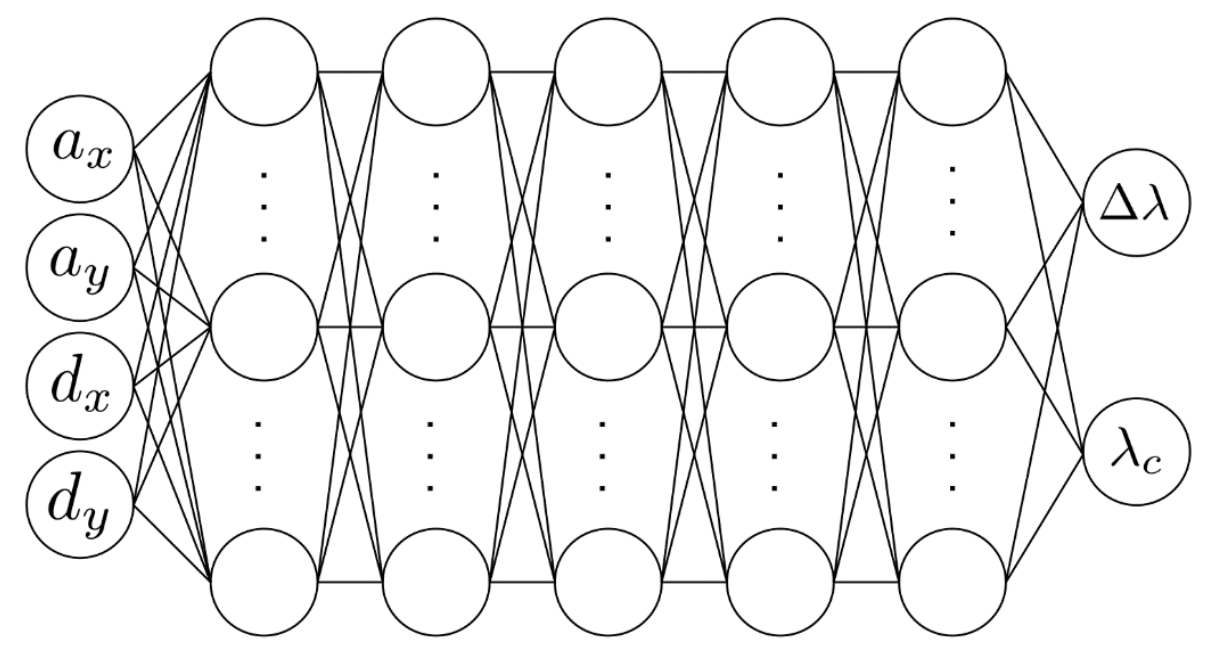

Figure 8. ANN architecture to predict the optical response for the rectangular lattice.

For the rectangular lattice, the input features are $a_{x}, a_{y}, d_{x}$ and $d_{y}$. Figure 8 shows the ANN architecture for the rectangular lattice. The architecture of the hidden layers of this ANN is the same as for the previous one. 
This ANN was trained with 10416 samples, split into $70 \%, 15 \%$, and $15 \%$ for training, testing, and cross-validation, respectively. In this case, the same optimizer was used, but with a learning rate of 0.01. Again, the loss function was mean square error, and the metric used was accuracy. The batch size was 500 and the number of epochs was 500 .

Using both ANNs, more data sets of optical response data were generated as a function of crystal characteristics. With the new data sets, further ANNs were trained to develop the inverse-design of the photonic crystals.

With this, the crystal characteristics were predicted from the optical response. In this case, the input features are the optical responses, that is $\Delta \lambda$ and $\lambda_{c}$. The output are the characteristics of the plasmonic crystal, $a$ and $f$ for the triangular lattice; and $a_{x}, a_{y}$ and $f$ for rectangular lattice.

The ANNs architectures for predicting crystal parameters from the optical response of the triangular and rectangular lattices are shown in Figs. 9 and 10, respectively. Both ANNs have 15 hidden layers, each with 500 neurons.

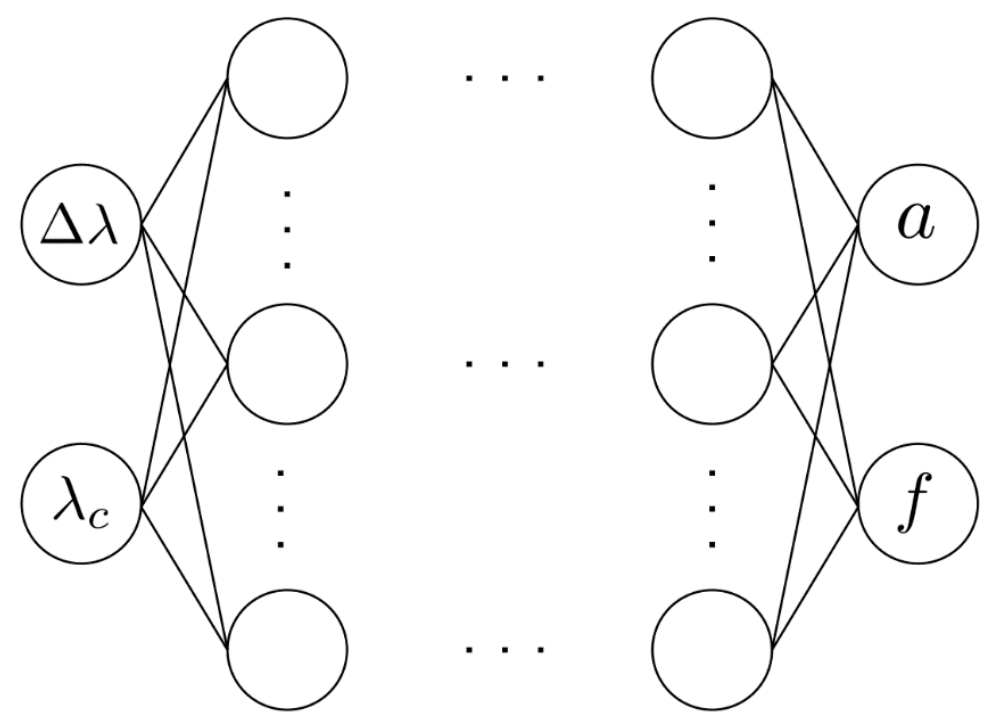

Figure 9. ANN architecture to predict the crystal parameters of a triangular lattice.

To train the ANN, 12126 samples, split into 70\%, 15\%, and 15\% were used for training, testing, and cross-validation, respectively. In addition, an Adam optimizer with a learning rate of 0.003 was used. The loss function and metric used were mean square error and cosine similarity, respectively. In this case, a batch size of 4000 and 400 epochs were used to train the algorithm.

On the other hand, the ANN for the rectangular lattice was trained with 262600 samples split in $90 \%, 5 \%$, and $5 \%$ for train, test, and cross-validation, respectively. The optimizer used was Adam with a learning rate of 0.0015. As above, the loss function and metric used were mean square error and cosine similarity, respectively. Finally, a batch size of 10000 and 100 epochs were used.

The ANNs were implemented using Keras with the TensorFlow backend. All hyperparameters were chosen for maximum accuracy. Also, to avoid underfitting and 
improve the ANN training performance, it was noted that the only geometry needed to be considered were bases with circular and square shapes for the triangular lattice and

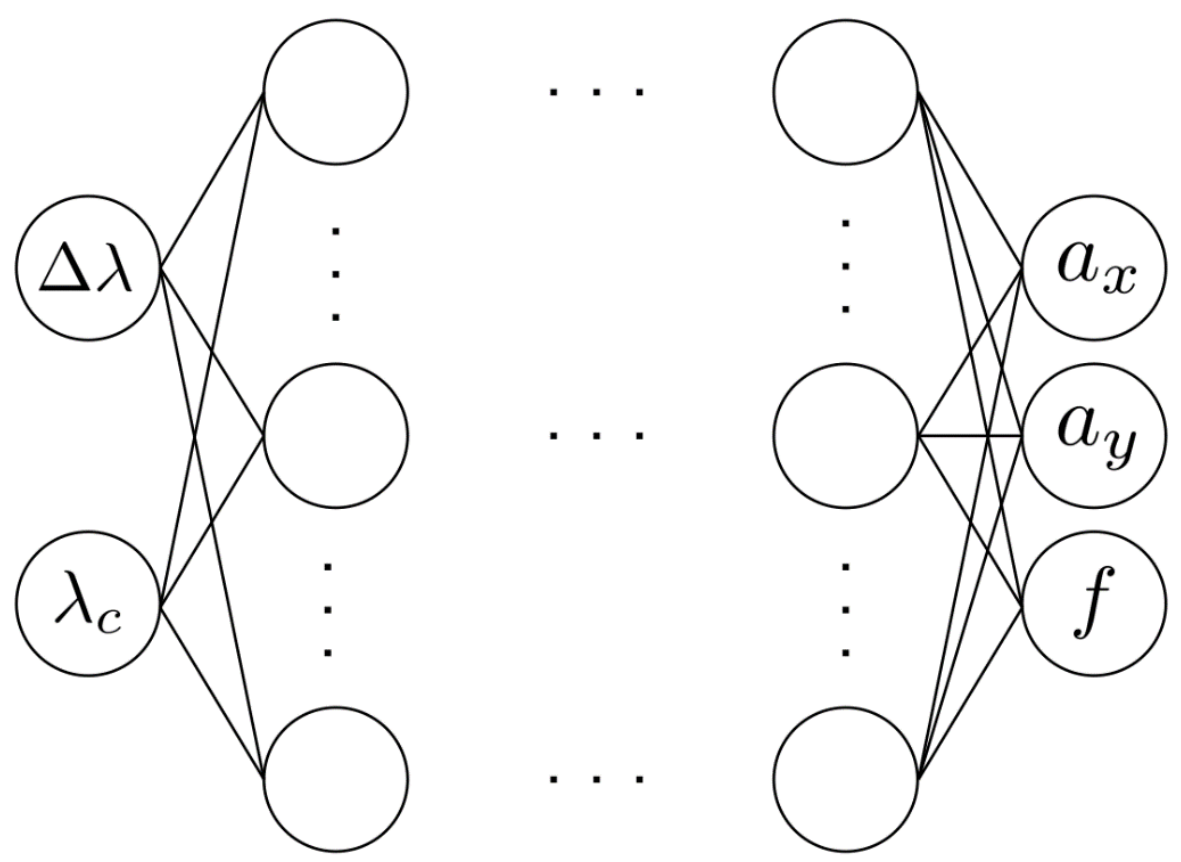

Figure 10. ANN architecture to predict the crystal parameters of a rectangular lattice.

elliptical, and rectangular shapes for the rectangular lattice.

\section{Results}

\subsection{One-dimensional PhPl crystal}

The one-dimensional PhPl crystal formed by layers of air and PMMA on a gold or silver thin film, as shown in Fig. 11.

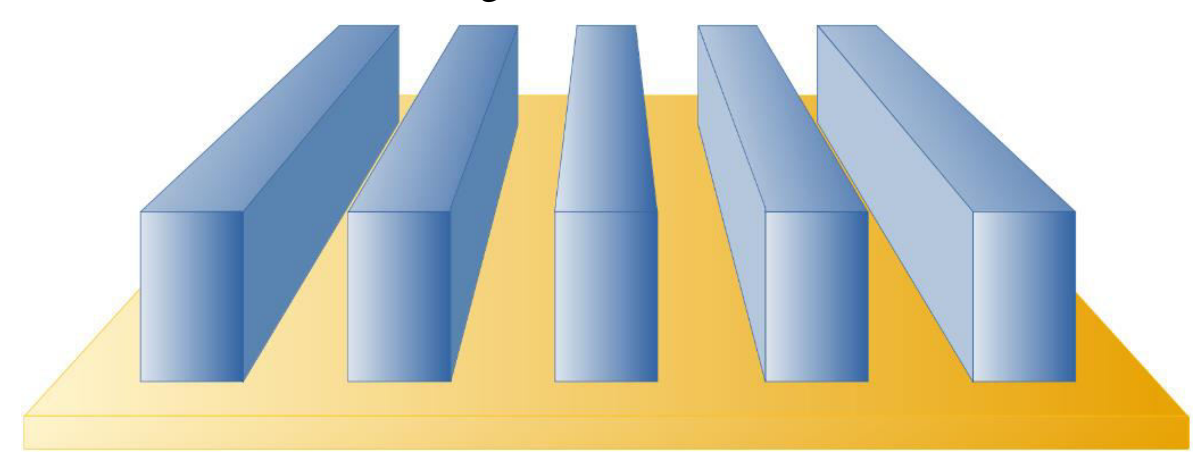

Figure 11. The one-dimensional PhPl crystal formed by stacks of air and PMMA on a thin metallic film. 
Figure 12 shows the band structure of a one-dimensional dielectric-gold PhPl crystal. The lattice constant is $a=300 \mathrm{~nm}$ and the filling fraction is $f=0.5$. With these parameters, it has a bandgap width, $\Delta \lambda=179.74 \mathrm{~nm}$, centered at $\lambda_{c}=787.22 \mathrm{~nm}$. The bandgap is represented with a gray fringe in the band structure.

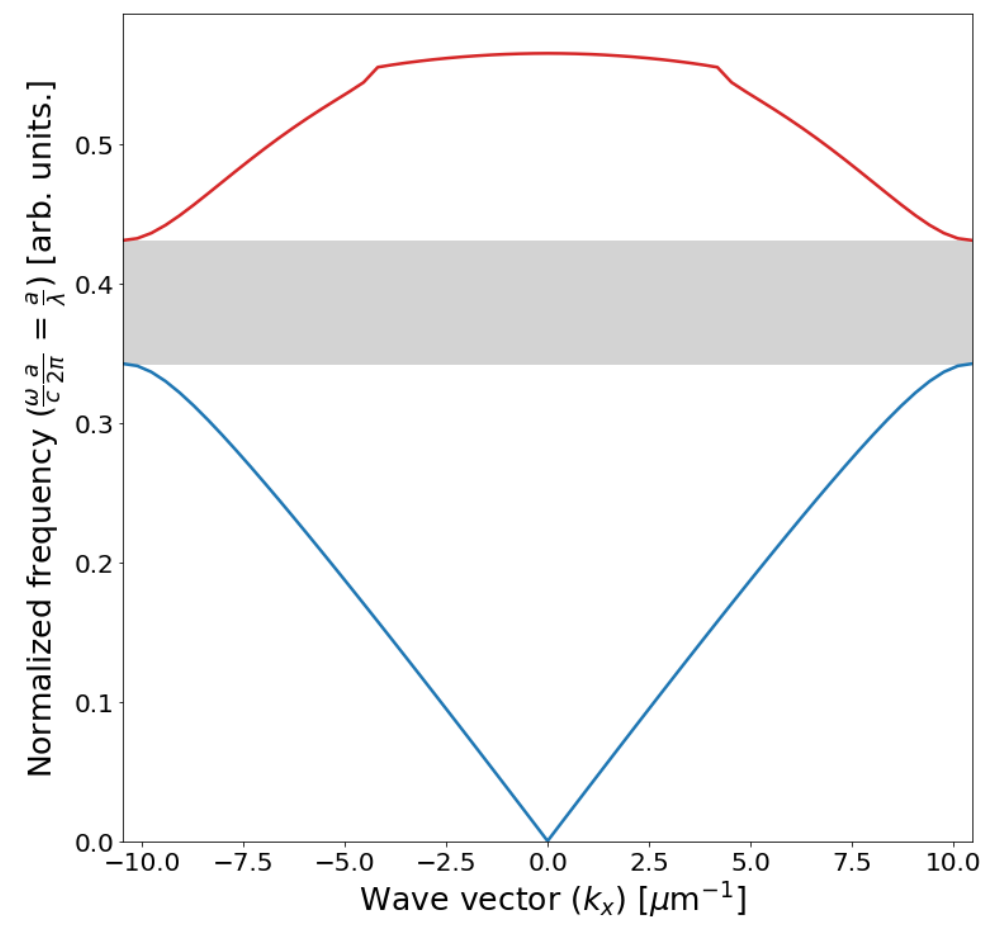

Figure 12. Band structure of a one-dimensional photonic crystal for $a=300 \mathrm{~nm}$ and $f=0.5$.

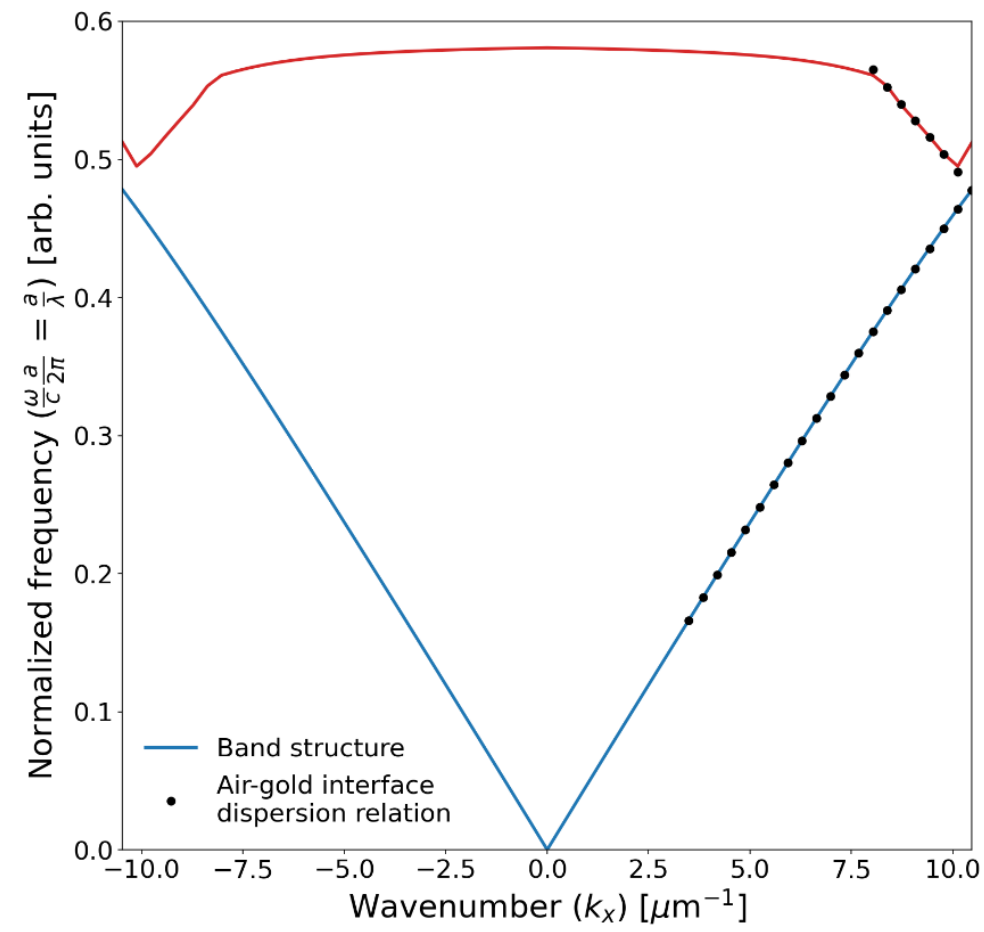

Figure 13. Band structure of a one-dimensional photonic crystal for $a=300 \mathrm{~nm}$ and $f=0$. Au case. 
To test the validity of the model, the band structure of a one-dimensional photonic crystal with two different filling fractions was calculated. The first value is $f=0$, which corresponds to a pure air-gold interface. The agreement shown in Fig. 13 between the band structure of the photonic crystal for $f=0$ and the dispersion relation of the SPP in an airgold interface, represented by the gray points, is evident.

On the contrary, for $f=1$, the physical system is a PMMA-gold interface, since the unit cell is completely covered by the PMMA layer. Figure 14 shows the corresponding

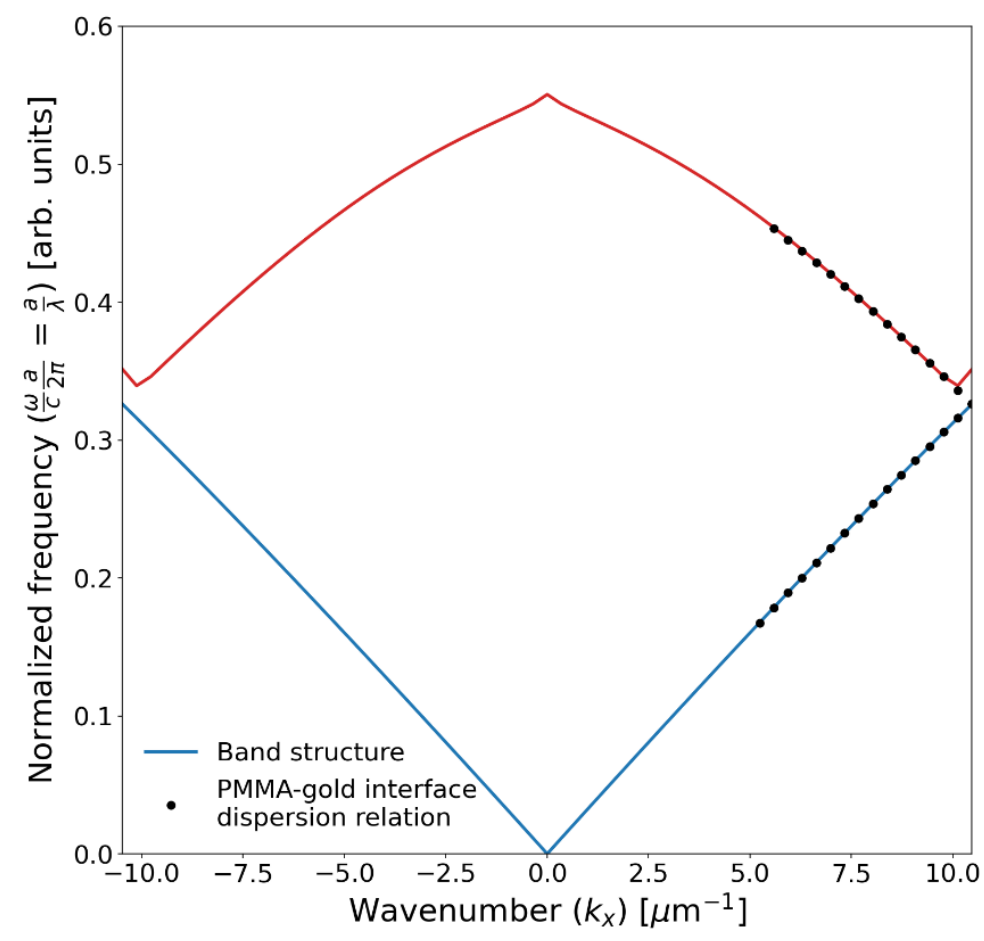

Figure 14. Band structure of a one-dimensional photonic crystal for $a=300 \mathrm{~nm}$ and $f=1$. Au case.

band structure. As above, the blue line is the band structure of the photonic crystal, while the gray points are the dispersion relation of the SPP in a PMMA-gold interface. Again, there is a total agreement among the band structure and the dispersion relation.

For a PhPl crystal with a lattice constant of $a=300 \mathrm{~nm}$ and a filling fraction $f=0.5$, but composed of a PMMA structure supported on a silver thin film, the photonic band structure is shown in Fig. 15. In this case, the center and width of the bandgap are $\lambda=782.09 \mathrm{~nm}$ and $\Delta \lambda=181.78 \mathrm{~nm}$, respectively.

Comparing the dielectric-silver PhPl crystal with the dielectric-gold $\mathrm{PhPl}$ one, there is a small difference between the bandgap characteristics of both when using the same parameters. This is because the effective indexes of refraction of a dielectric-gold interface and a dielectric-silver interface are similar in the electromagnetic spectrum where the bandgap arises.

To understand how the optical response is modified as a function of crystal parameters, the band structure was calculated for different lattice constants and filling 


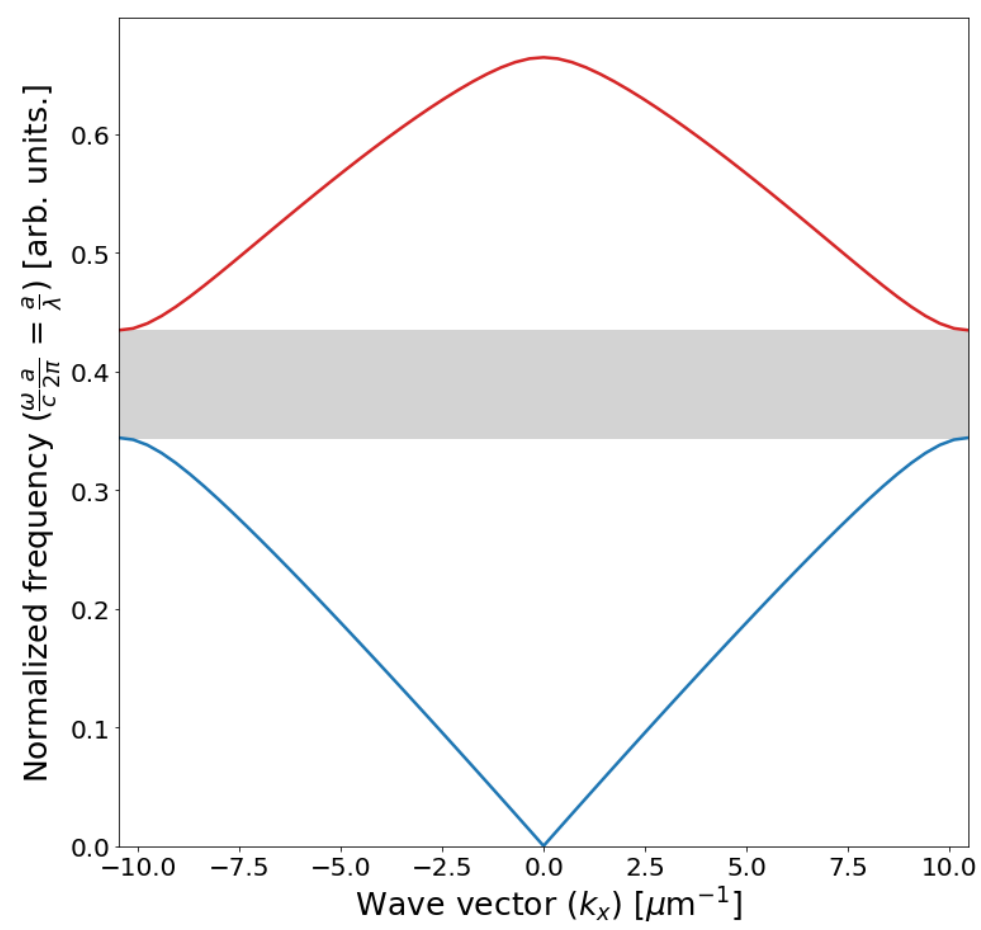

Figure 15. Band structure of a one-dimensional photonic crystal for $a=300 \mathrm{~nm}$ and $f=0.5$. Ag case.

fractions for a dielectric-gold $\mathrm{PhPl}$ crystal. Figure 16 shows the behavior of the bandgap center as a function of lattice constant and filling fraction. As it is shown in the plot, the bandgap center increases monotonically as the lattice constant and filling fraction do too.

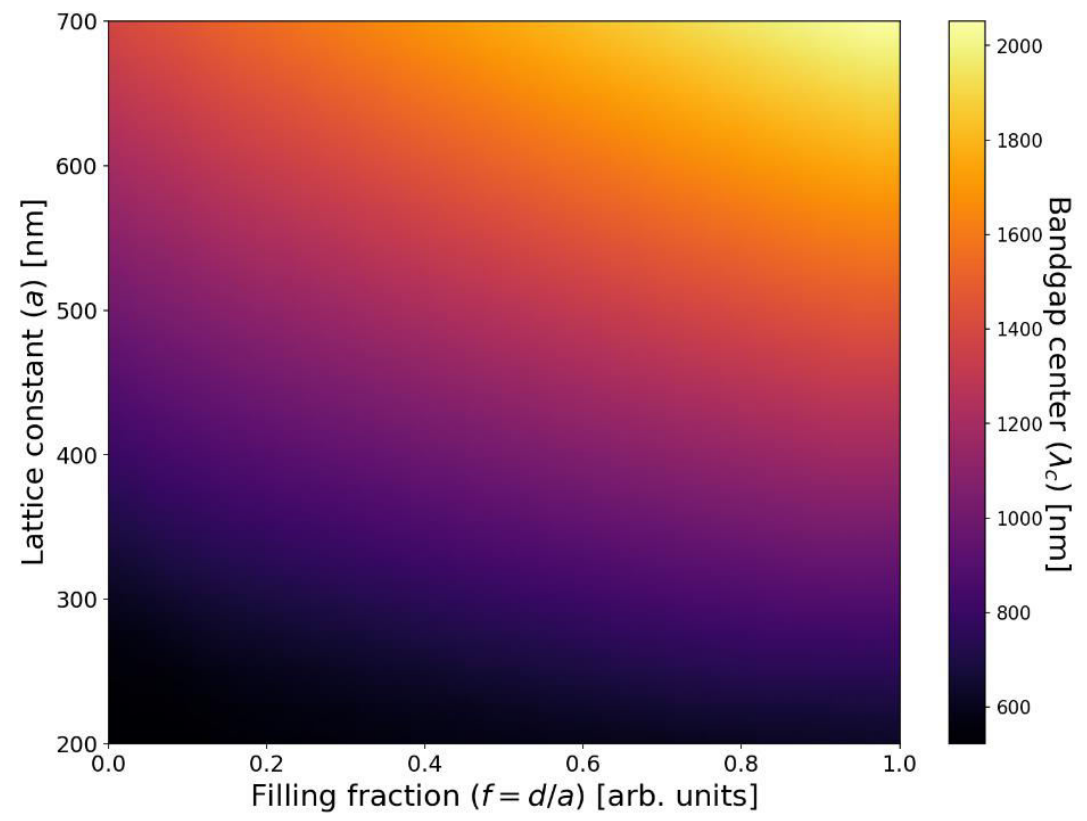

Figure 16. Bandgap center as a function of $a$ and $f$ for onedimensional dielectric-gold PhPl crystal. 
On the other hand, the bandgap width as a function of the lattice constant and filling fraction is shown in Figure 17. In this case, $\Delta \lambda$ increases as the lattice constant does. However, as a function of the filling fraction, it increases until it reaches a maximum near $f=0.44$, and then it decreases to zero.

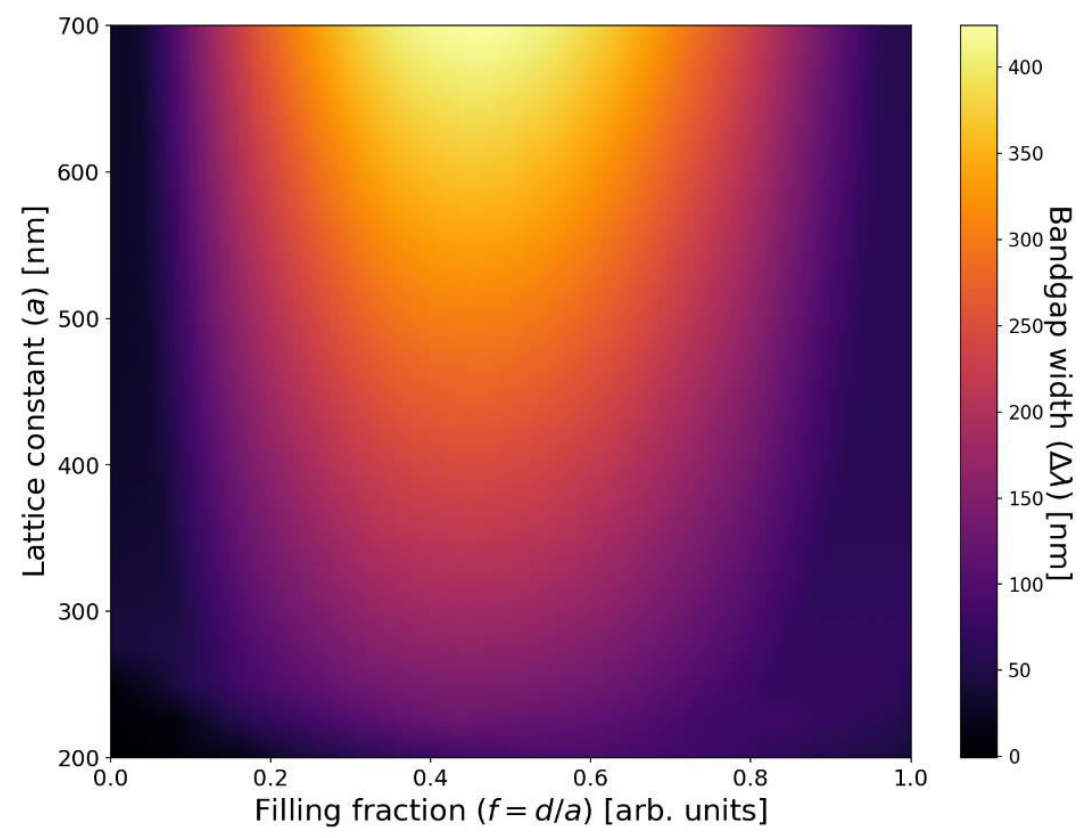

Figure 17. Bandgap width as a function of $a$ and $f$ for onedimensional dielectric-gold PhPl crystal.

\subsection{Two-dimensional PhPl crystals}

As it was mentioned above, three different two-dimensional lattices were analyzed. For each case, the optical response (the bandgap width $\Delta \lambda$ and center $\lambda_{c}$ ) was studied as a function of the lattice constant $a$, and the base sizes $d_{x}$ and $d_{y}$. Then, an ANN was trained to predict the photonic crystal parameters for the desired optical response. For all the cases, numerical simulations were obtained to corroborate the analytical results. The simulations are shown only for the triangular and the rectangular lattices.

\subsubsection{Square lattice}

For a square lattice with a $300 \mathrm{~nm}$ lattice constant and an elliptical base with $165 \mathrm{~nm}$ for the major axis, and $132 \mathrm{~nm}$ for the minor one, the band structure is shown in Fig. 18. In this case, the major axis is in the $x$ direction, while the minor axis is in the $y$ direction. For the square lattice, there are two partial bandgaps. The first is on the $\Gamma-\mathrm{X}$ path, which has a width of $90.30 \mathrm{~nm}$ and is centered at $696.19 \mathrm{~nm}$. The second is on the $\mathrm{M}-\Gamma$ path, with a width of $72.55 \mathrm{~nm}$ and center at $573.64 \mathrm{~nm}$.

Note that bands 2 and 3 (black and red curves, respectively) coincide at the $\Gamma$ point with a value of 0.57 , which is equivalent to a wavelength of about $526.32 \mathrm{~nm}$. Moreover, the upper bands cluster near this same value, that is, near the fitting parameter $b$ of 


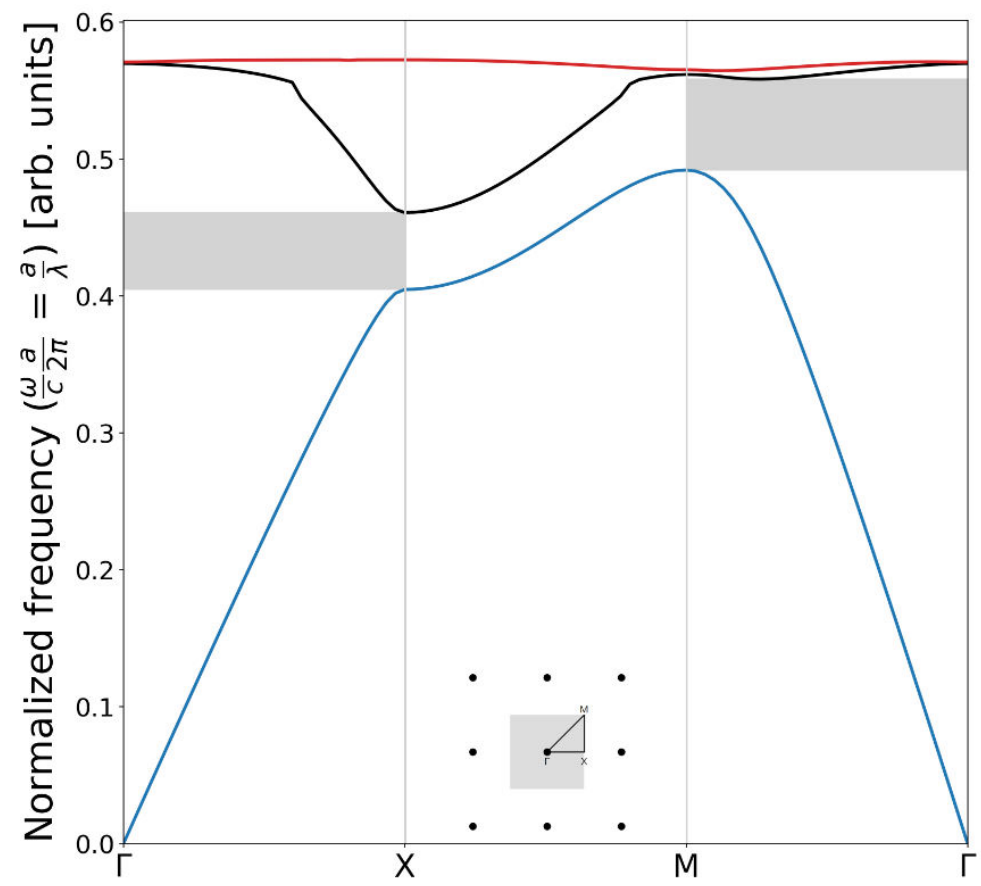

Figure 18. Band structure of a dielectric-gold PhPl crystal for a square lattice with an elliptical base. This band structure corresponds to a lattice constant of $a=300$ $\mathrm{nm}$, with $d_{x}=165 \mathrm{~nm}$ and $d_{y}=132 \mathrm{~nm}$.

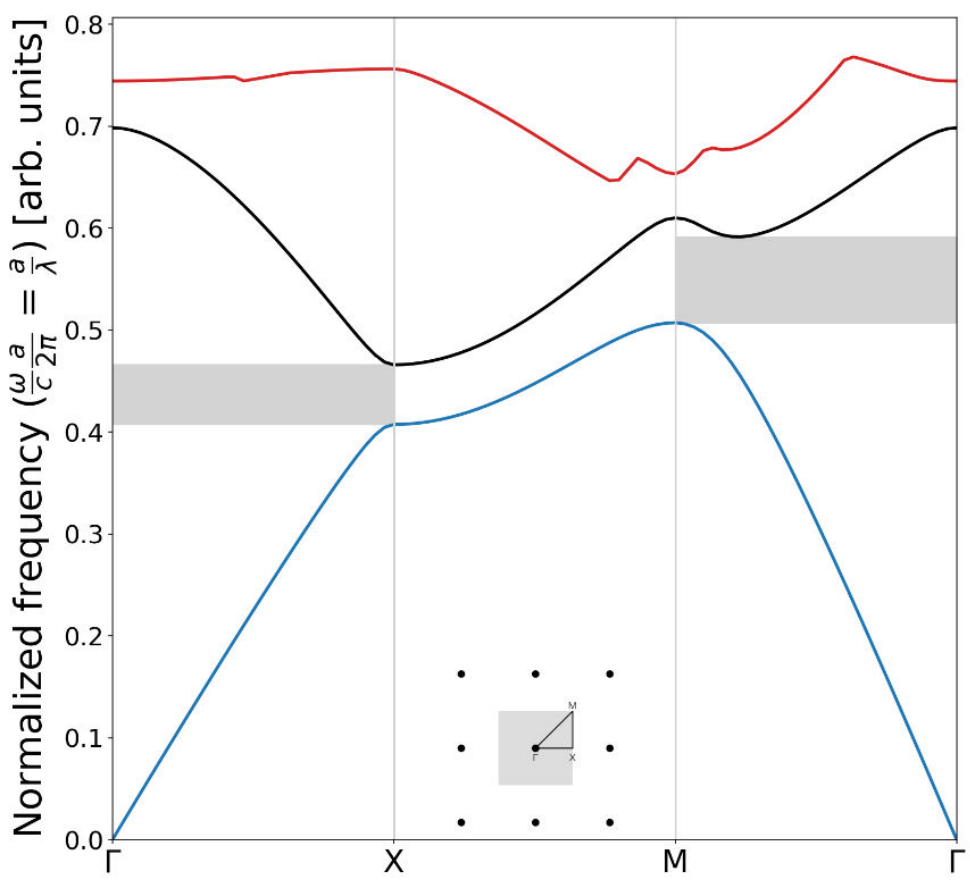

Figure 19. Band structure of a dielectric-silver $\mathrm{PhPl}$ crystal for a square lattice with an elliptical base. This band structure corresponds to a lattice constant of $a=300 \mathrm{~nm}, d_{x}=165 \mathrm{~nm}$ and $d_{y}=132 \mathrm{~nm}$. 
equations (13) and (14) for a dielectric-gold interface. This means that the bandgap is within the wavelength range in which the data fitting was made.

Using the same parameters as above, the band structure of a dielectric-silver $\mathrm{PhPl}$ crystal is shown in Fig. 19. The bandgap in the $\Gamma-\mathrm{X}$ path is centered at $689.94 \mathrm{~nm}$ and has a width of $92.37 \mathrm{~nm}$. The bandgap in the $\mathrm{M}-\Gamma$ path is centered at $549.22 \mathrm{~nm}$ and has a width of $84.26 \mathrm{~nm}$.

In this case, the characteristics of the bandgap in the $\Gamma-\mathrm{X}$ path are similar to the dielectric-gold $\mathrm{PhPl}$ crystal. However, characteristics of both bandgaps in the $\mathrm{M}-\Gamma$ path are remarkably different. This is due to the difference of the effective indexes of refraction in that range of the electromagnetic spectrum.

As in the previous case, for a dielectric-silver PhPl crystal, the upper bands cluster near parameter $b$ of the fitting equation. This means that the dielectric-silver PhPl crystal can be used over a wider range of wavelengths.

On the other hand, the reason of the existence of two partial bandgaps is a low contrast of dielectric constants. For example, in a photonic crystal with the same dimensions but with a dielectric constants contrast of $\epsilon_{b} / \epsilon_{a}=9$, there are two complete bandgaps, as it is shown in Fig. 20.

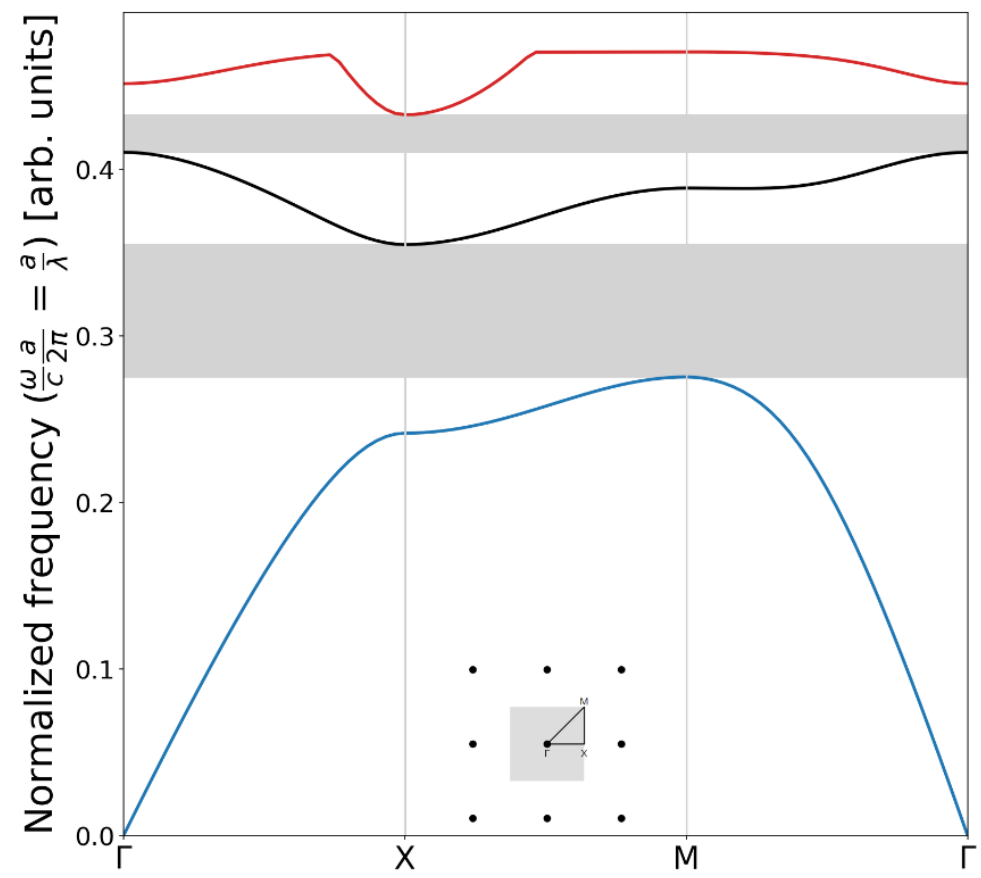

Figure 20. Band structure of a square lattice with an elliptical base. This band structure corresponds to a lattice constant of $a=300 \mathrm{~nm}, d_{x}=165 \mathrm{~nm}$ and $d_{y}=132$ $\mathrm{nm}$, but with a dielectric constants contrast of $\epsilon_{b} / \epsilon_{a}=9$. 
In this case, one of the bandgaps has a width of $\Delta \lambda=243.76 \mathrm{~nm}$ and is centered at $\lambda_{c}=966.93 \mathrm{~nm}$. The other bandgap has a width of $\Delta \lambda=38.22 \mathrm{~nm}$ and is centered at $\lambda_{c}=711.9 \mathrm{~nm}$. In addition, as the contrast of the dielectric constants increases, the width and center of the bandgap also increase.

As in the one-dimensional case, the center and width of the bandgap were calculated for a square crystal with rectangular and elliptical bases. As we could verify, the geometry of the base cross-section does not represent a significant difference in the band structure, the key parameter is the filling fraction.

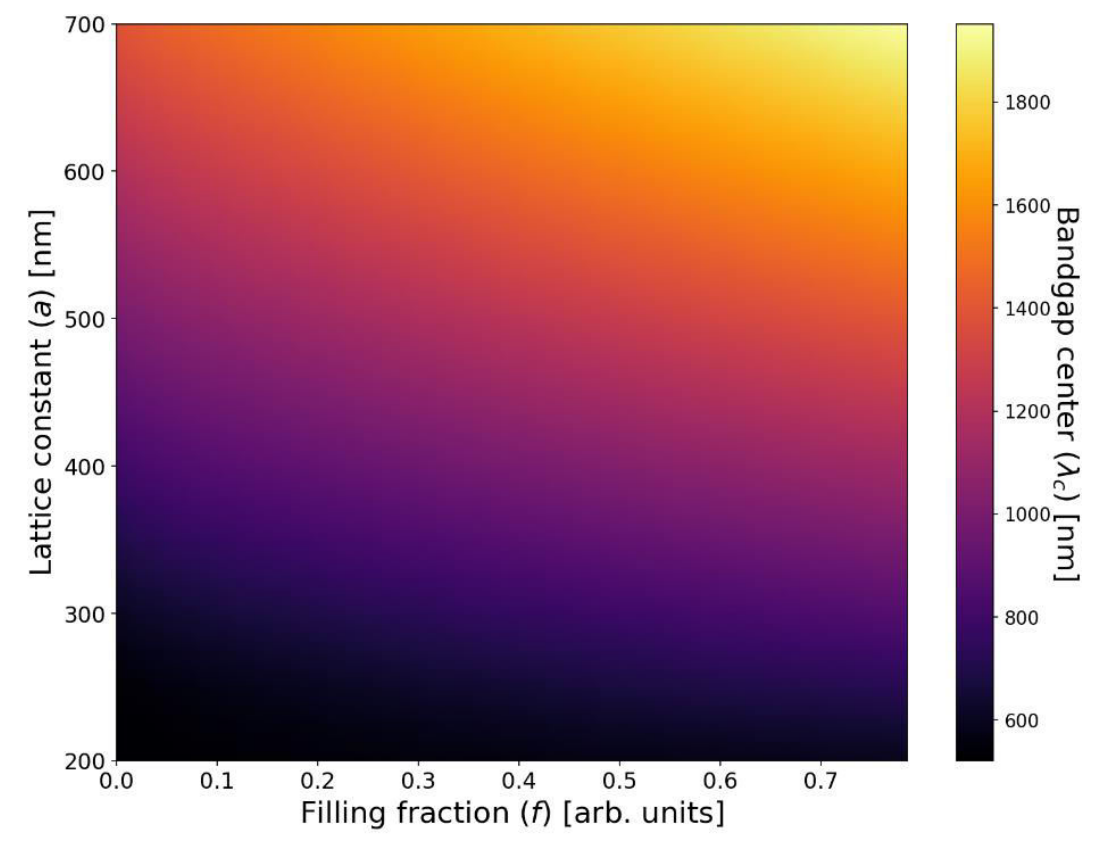

Figure 21. Bandgap center as a function of $a$ and $f$ for square lattice and with an elliptical base.

Figures 21 and 22 show heat maps of the center and width of the bandgap in the path $\Gamma$ $-\mathrm{X}$, respectively, as a function of the lattice constant $a$ and filling fraction $f$. As in the onedimensional case, the center of bandgap is an increasing function of the lattice constant and the filling fraction. Furthermore, the bandgap increases up to a maximum near $f=0.38$, decreasing after that.

Having the above into account, to tune the bandgap center and width for a square lattice, it is necessary adjusting the lattice constant and filling fraction. Furthermore, it is possible obtaining a complete bandgap, by varying the dielectric constants contrast. 


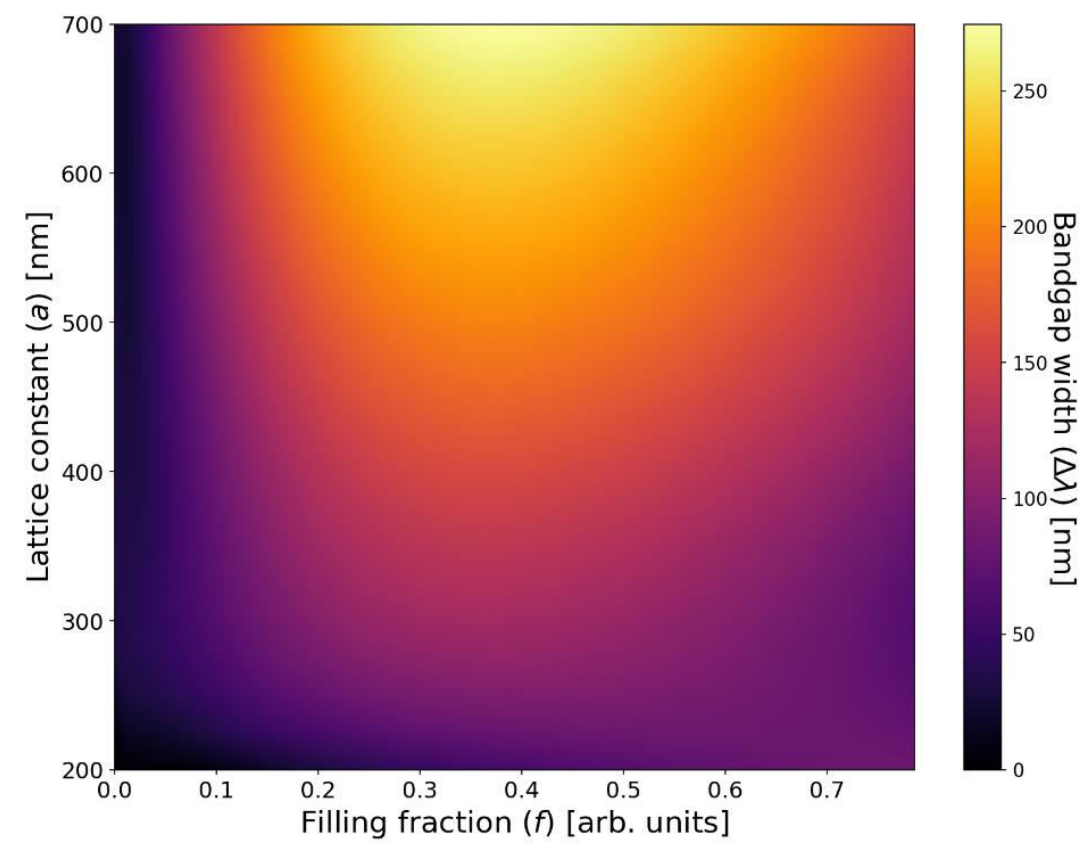

Figure 22. Bandgap width as a function of $a$ and $f$ for square lattice and with an elliptical base.

\subsubsection{Triangular lattice}

Unlike the square lattice, the triangular lattice presents a complete bandgap even at low dielectric constants contrast, as it is shown in Fig. 23.

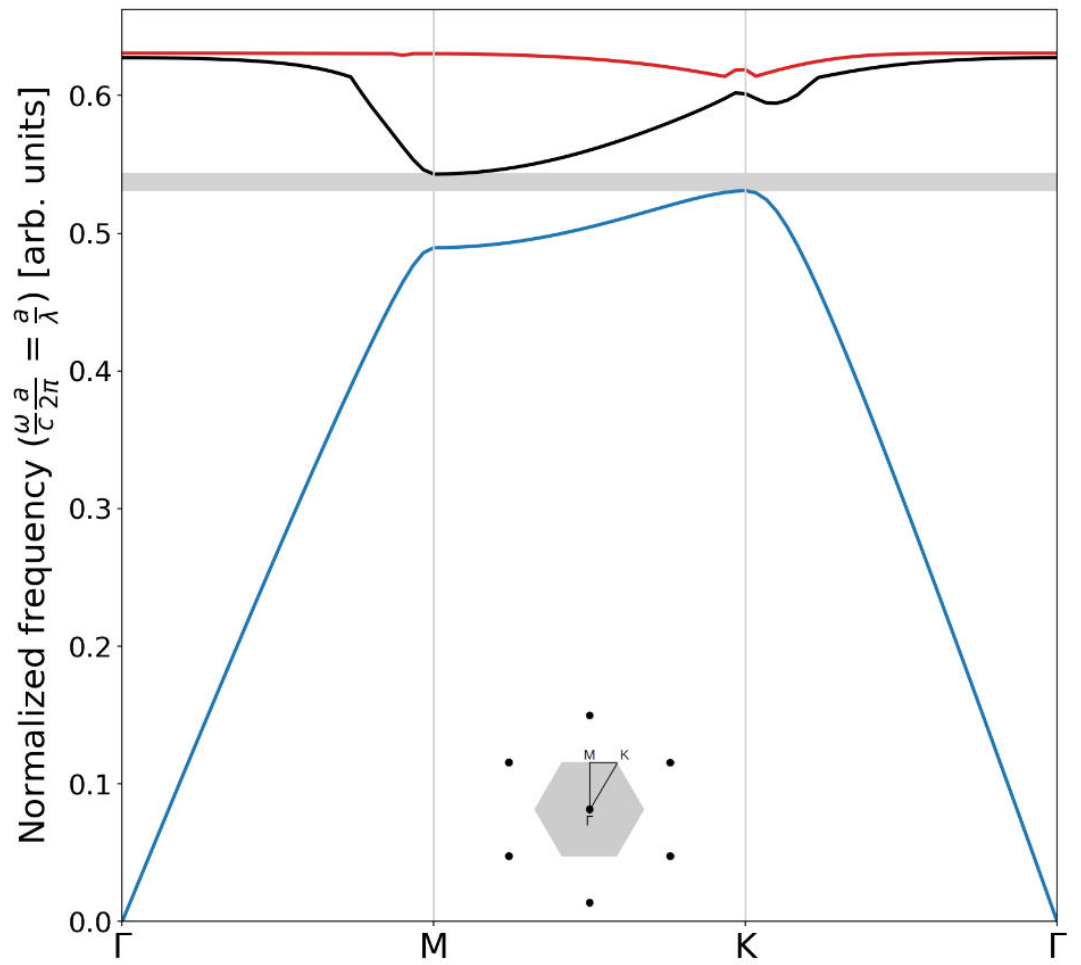

Figure 23. Band structure of a dielectric-gold PhPl crystal for a triangular lattice with an elliptical base, with $a=330 \mathrm{~nm}$, $d_{x}=130 \mathrm{~nm}$ and $d_{y}=100 \mathrm{~nm}$. 
This band structure corresponds to a lattice constant of $330 \mathrm{~nm}$, with an elliptical base whose major axis is $d_{x}=130 \mathrm{~nm}$, while the minor axis is $d_{y}=100 \mathrm{~nm}$. The bandgap has a width of $42.82 \mathrm{~nm}$ and is centered at $\lambda_{c}=631.33 \mathrm{~nm}$.

The photonic band structure of a dielectric-silver PhPl crystal with the same lattice constant and base size is shown in Fig. 24. The bandgap is centered at $\lambda_{c}=620.16 \mathrm{~nm}$ with a width of $\Delta \lambda=40.98 \mathrm{~nm}$.

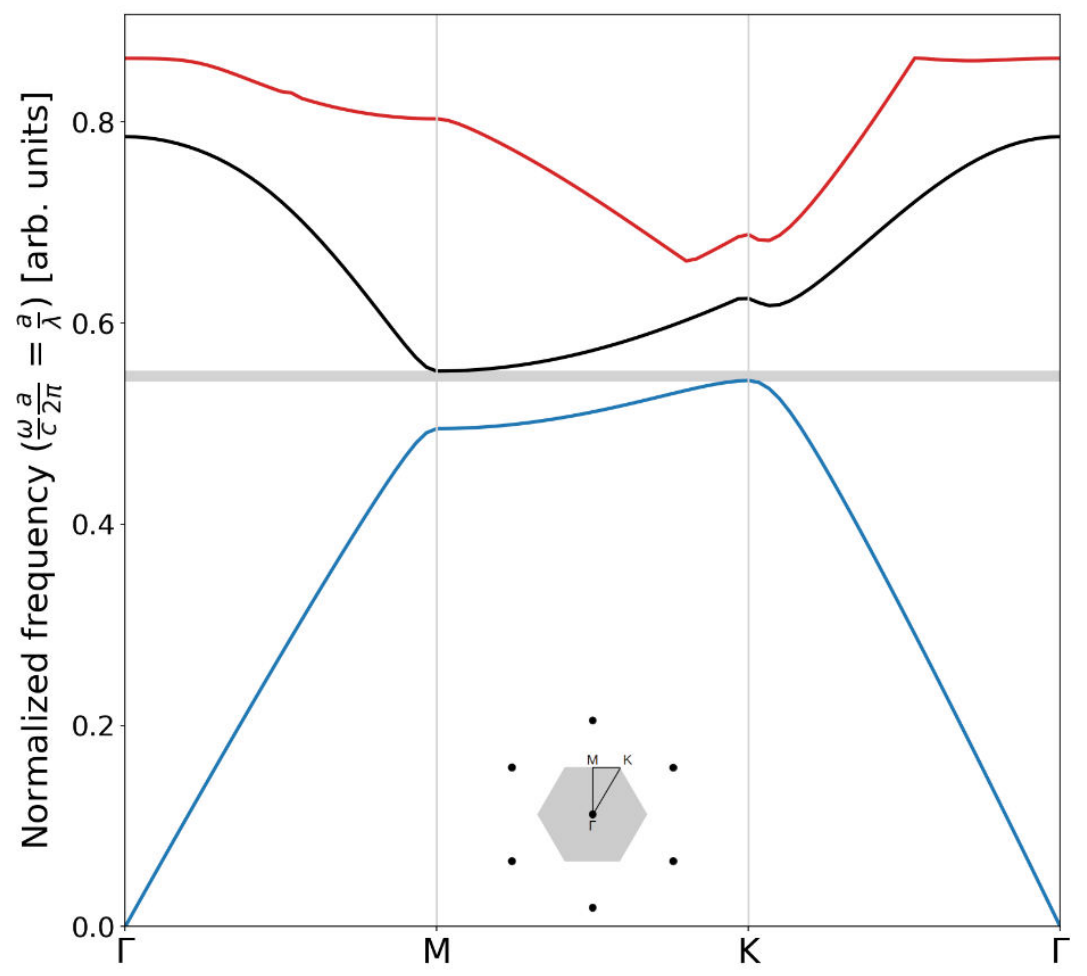

Figure 24. Band structure of a dielectric-silver PIPh crystal for a triangular lattice with an elliptical base, with $a=330 \mathrm{~nm}$, $d_{x}=130 \mathrm{~nm}$ and $d_{y}=100 \mathrm{~nm}$.

Comparing this $\mathrm{PhPl}$ crystal with a dielectric-gold $\mathrm{PhPl}$ crystal, the significant change is in the bandgap center. The difference between both bandgap centers is about $11 \mathrm{~nm}$.

Figure 25 shows the bandgap width as a function of lattice size and filling fraction. In this case, a complete bandgap arises for filling fractions larger than 0.3 and less than 0.85 and reaches its maximum near $f=0.44$. Furthermore, the bandgap width is a monotonically increasing function of the lattice constant. For the wavelength interval used, the maximum width is $\Delta \lambda=99.56 \mathrm{~nm}$.

With respect to the ANN, an accuracy of 1 is obtained to predict the optical response as a function of plasmonic crystal characteristics. As an example, with a lattice constant of $a=300 \mathrm{~nm}$ and ratios $f_{x}=d_{x} / a=0.6$ and $r_{y}=d_{y} / a=0.8$, the ANN predicts a bandgap width of $\Delta \lambda=22.46 \mathrm{~nm}$, centered at $\lambda_{c}=663.04 \mathrm{~nm}$. With the same values, according with the analytical model, the width and center of gap are $\Delta \lambda=22.75 \mathrm{~nm}$ and $\lambda_{c}=661.95 \mathrm{~nm}$, respectively. 


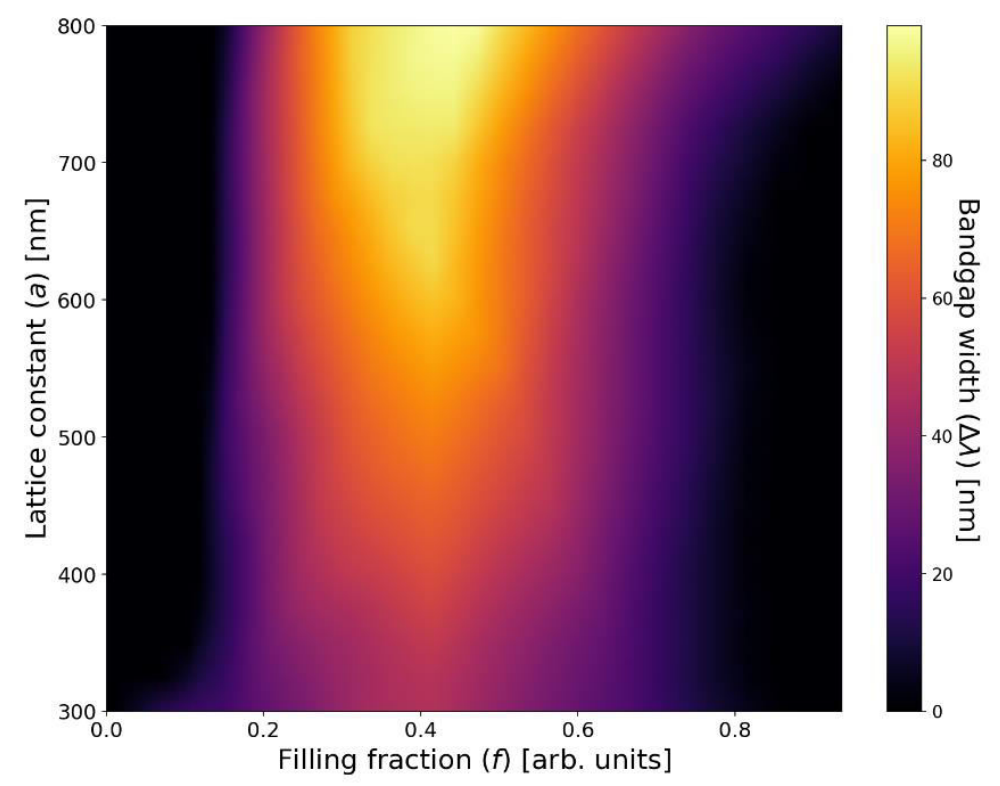

Figure 25. Bandgap width as a function of $a$ and $f$ for triangular lattice and with an elliptical base.

For the inverse design, the trained ANN has a cosine similarity of 0.988 to predict the plasmonic crystal characteristics for a particular optical response. As an example, for a bandgap width of $\Delta \lambda=20 \mathrm{~nm}$, centered at $\lambda_{c}=633 \mathrm{~nm}$, the ANN predicts a lattice constant of $a=328.32 \mathrm{~nm}$, and a filling fraction of $f=0.1287938$. This filling fraction corresponds to a circular base with diameter value of $d=123.72 \mathrm{~nm}$.

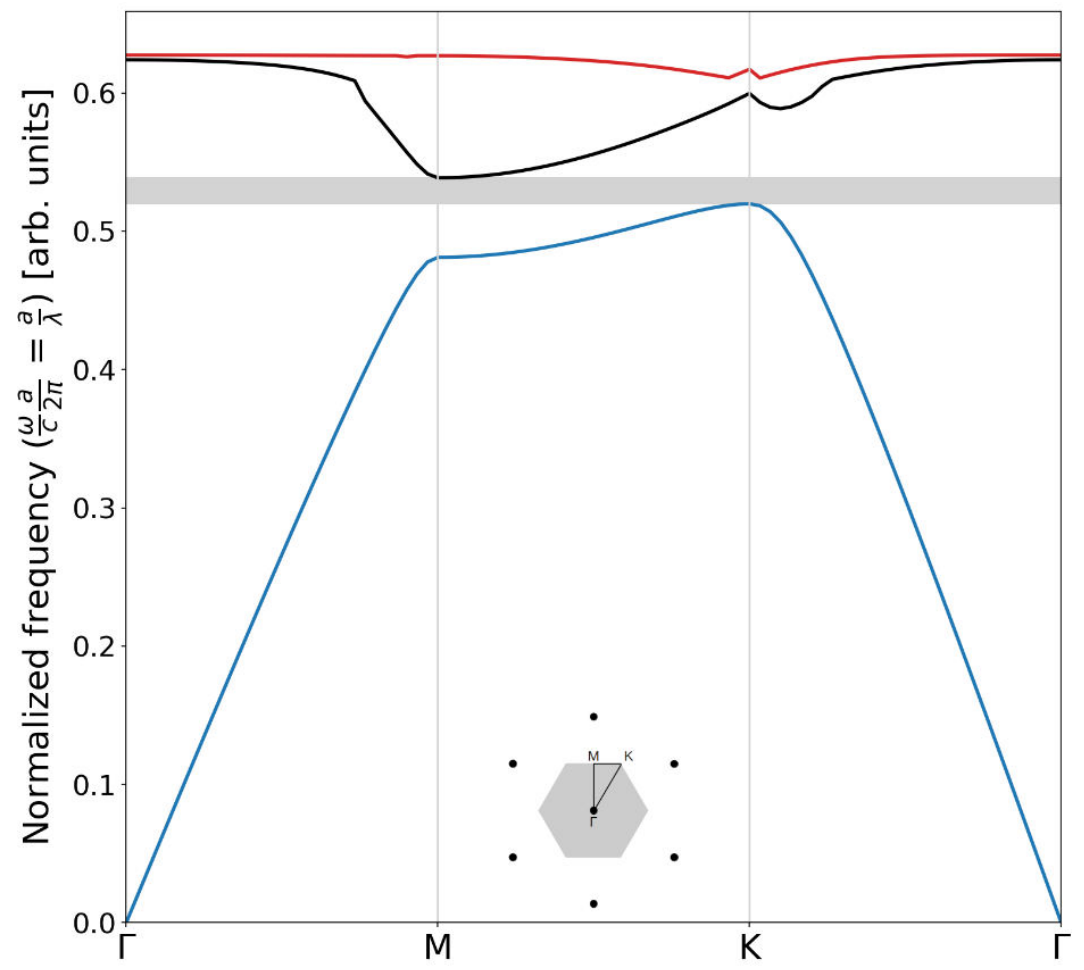

Figure 26. Band structure of a dielectric-gold PhPl crystal for a triangular lattice with a circular base, with $a=328.32 \mathrm{~nm}$ and $d=123.72 \mathrm{~nm}$. 
Using the data provided for the ANN, the bandgap width is $\Delta \lambda=22.09 \mathrm{~nm}$ and is centered at $\lambda_{c}=620.61 \mathrm{~nm}$. Figure 26 shows the photonic band structure of this crystal. In this case, the difference between the predicted and the actual bandgap width is $2.09 \mathrm{~nm}$. Concerning the bandgap centers, the difference between predicted and the actual is 12.39 $\mathrm{nm}$.

To support both, the results obtained with the analytical model and those the ANNs, numerical simulations were performed using Comsol Multiphysics® software. It is worth mentioning that the numerical simulations were performed for all orientations, however, for illustrative purposes, only the numerical simulations for the $\mathrm{PhPl}$ crystal in the $\Gamma-\mathrm{M}$ orientation are shown.

Figure 27 shows a numerical simulation of an incident SPP at $\lambda=605 \mathrm{~nm}$ in a dielectric-gold PhPl crystal. This wavelength corresponds to an allowed frequency.

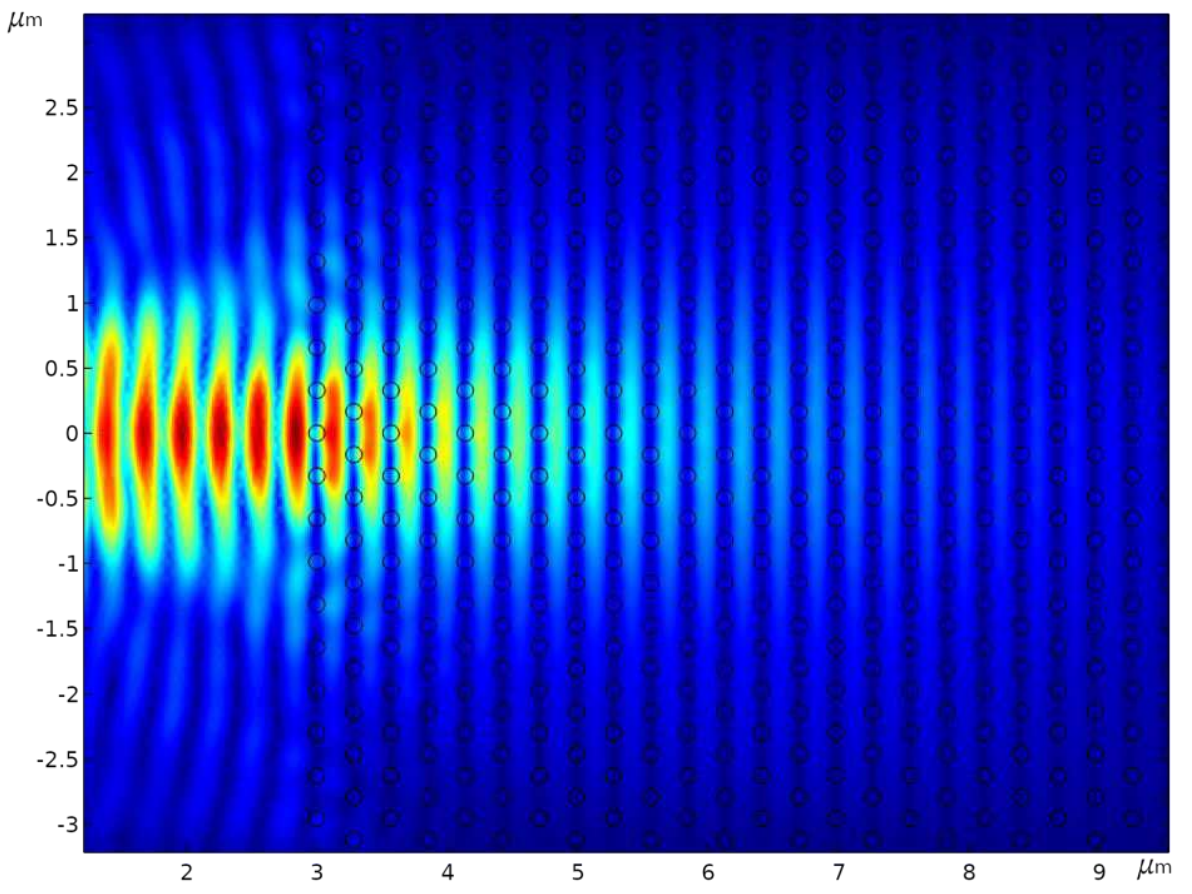

Figure 27. Numerical simulation of incident electric field at $\lambda=605 \mathrm{~nm}$ in a $\mathrm{PhPl}$ crystal, at an allowed frequency.

Figure 28 shows a numerical simulation of an incident electric field at $\lambda=633 \mathrm{~nm}$ in a $\mathrm{PhPl}$ crystal, that is, at a frequency within the photonic bandgap.

Figure 29 shows a numerical simulation of an incident electric field at $\lambda=690 \mathrm{~nm}$ in a PhPl crystal. This wavelength corresponds to an allowed frequency in the partial photonic bandgap in the $\Gamma-\mathrm{M}$ path.

The PhPl crystal has a lattice constant of $a=328.32 \mathrm{~nm}$, a base diameter $d=$ $123.72 \mathrm{~nm}$ and is oriented in the $\Gamma-\mathrm{M}$ path. 


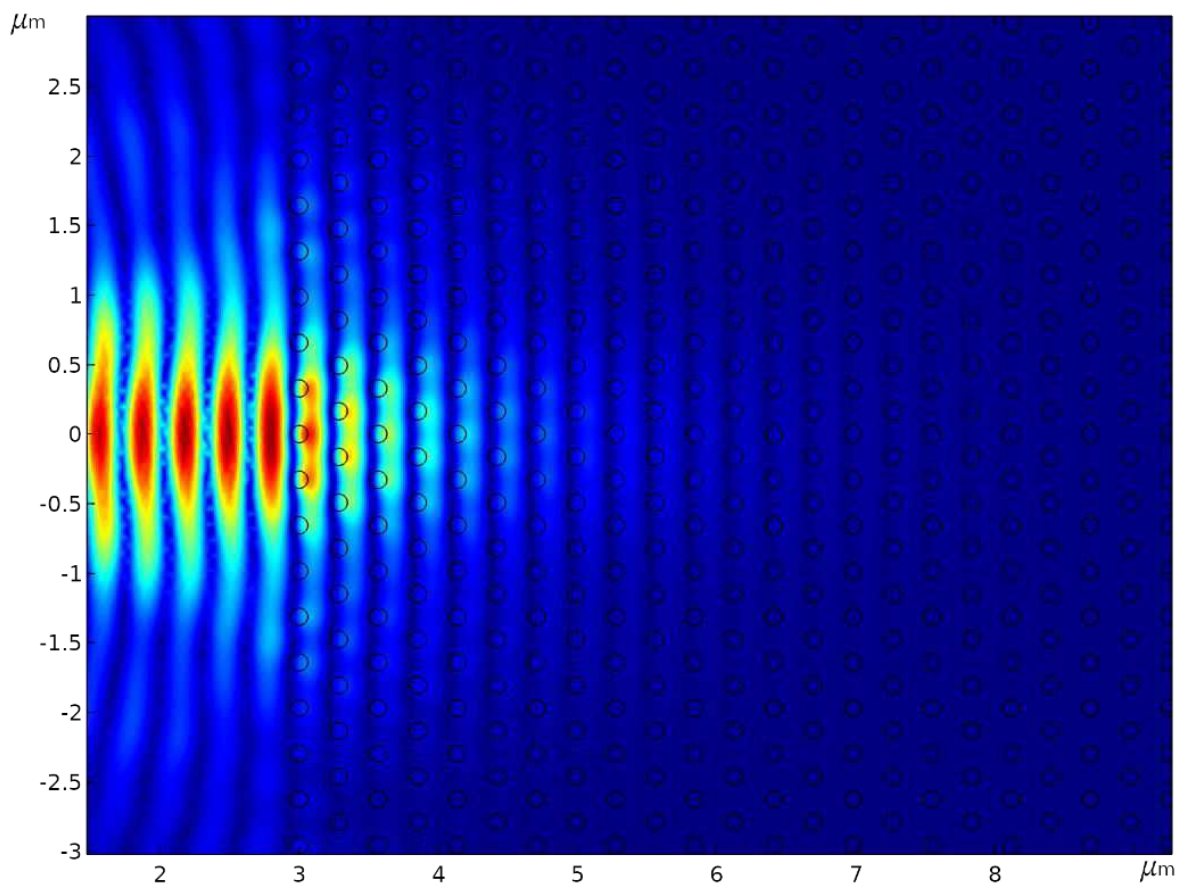

Figure 28. Numerical simulation of incident electric field at $\lambda=633 \mathrm{~nm}$ in a PhPl crystal, at a frequency within the bandgap.

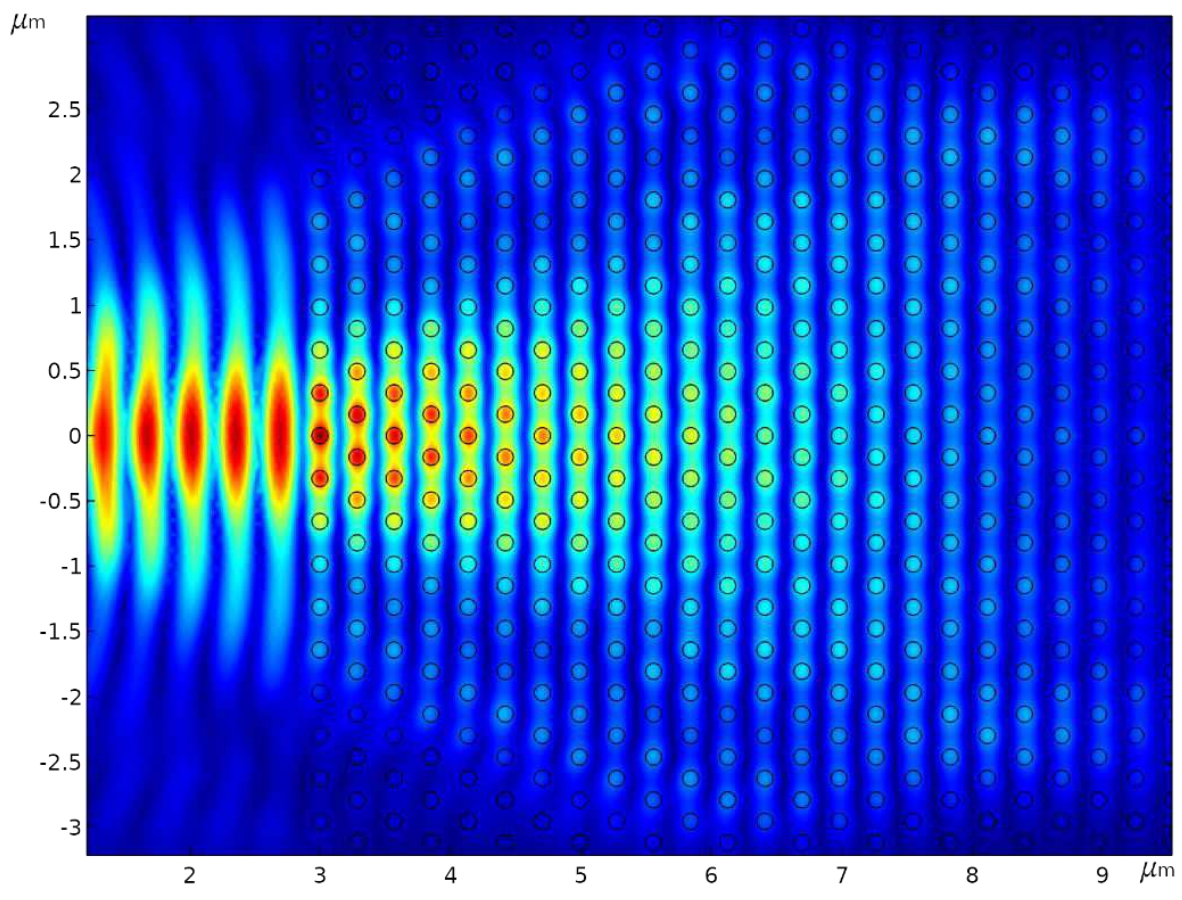

Figure 29. Numerical simulation of incident electric field at $\lambda=690 \mathrm{~nm}$ in a PhPl crystal, at an allowed frequency. 
The normalized absolute value of the electric field at $\lambda=633 \mathrm{~nm}$, that is, within the bandgap, is compared to the normalized absolute value of the electric field at the two allowed wavelengths in the $\Gamma-\mathrm{M}$ path, 605 and $690 \mathrm{~nm}$.

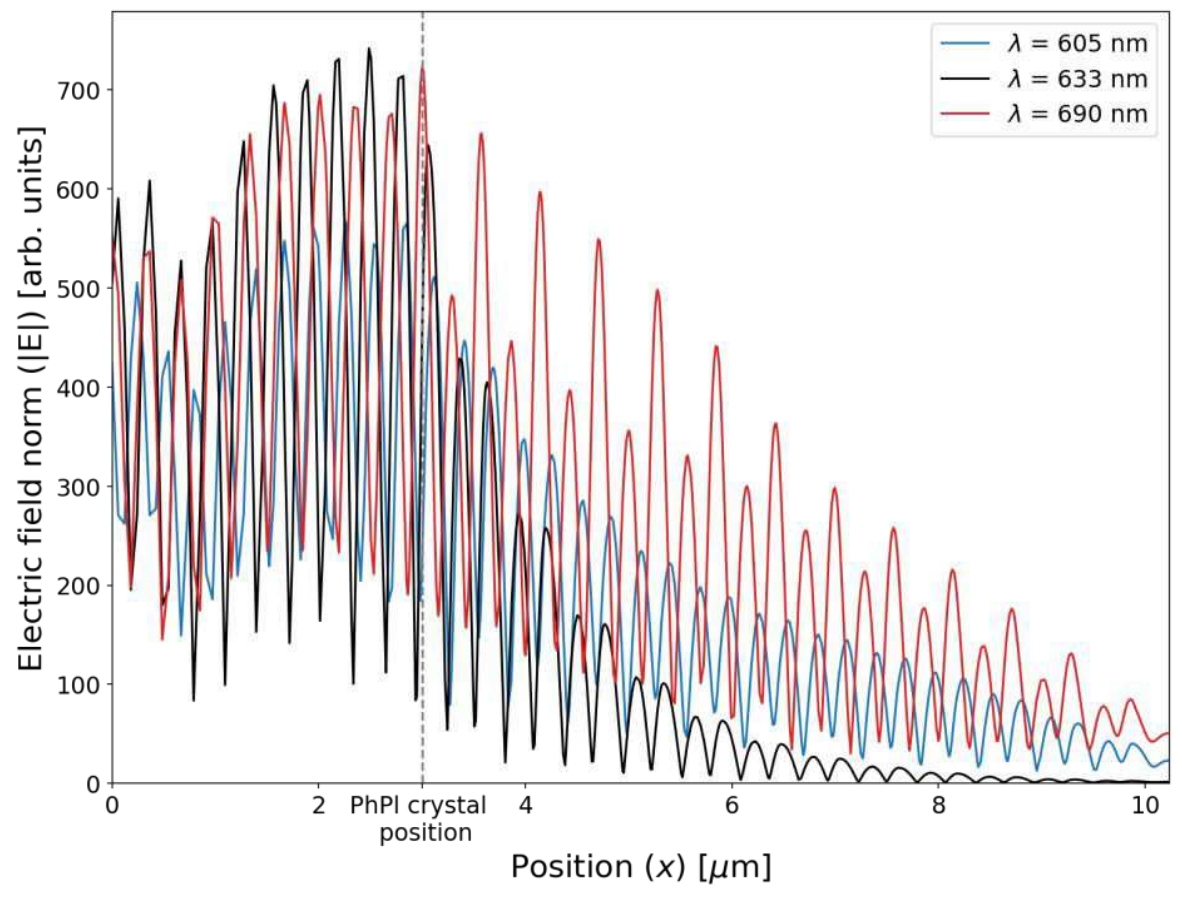

Figure 30. Comparison of the normalized absolute value of the electric field for three different wavelengths.

This is shown in Fig. 30, where the dashed gray line corresponds to $605 \mathrm{~nm}$, the dashed black line corresponds to $633 \mathrm{~nm}$ and the solid gray line corresponds to $690 \mathrm{~nm}$. The vertical dashed blue line marks where the PhPl crystal begins.

For all the three wavelengths, before entering to the PhPl crystal, the field intensity increases due to reflections on the PhPl crystal. After that, for all the three wavelengths, the electric field intensity, decreases as it enters the PhPl crystal, since SPPs intensity decays exponentially, as it is shown in the graph. However, the electric field corresponding to the wavelength within the bandgap, $633 \mathrm{~nm}$, decreases faster than the other wavelengths.

Although the imaginary part of the effective index of refraction at $605 \mathrm{~nm}$ is larger than the corresponding at $633 \mathrm{~nm}$, since the system has been tuned to present a bandgap at this last wavelength, the field intensity decreases faster for this case, corroborating the physics behind the proposed analytical model.

\subsubsection{Rectangular lattice}

In this case, the theoretical model was used to analyze the band structures of dielectric-gold and dielectric-silver PhPl crystals in a rectangular lattice, with elliptical and rectangular bases. 
Figure 31 shows the photonic band structure of a dielectric-gold $\mathrm{PhPl}$ in a rectangular lattice with a circular base, where this base is considered a particular case of an elliptical base. The lattice constant in $x$-direction is $a_{x}=300 \mathrm{~nm}$, in $y$-direction is $a_{y}=480 \mathrm{~nm}$ and the diameter of the cross-section is $d=235 \mathrm{~nm}$.

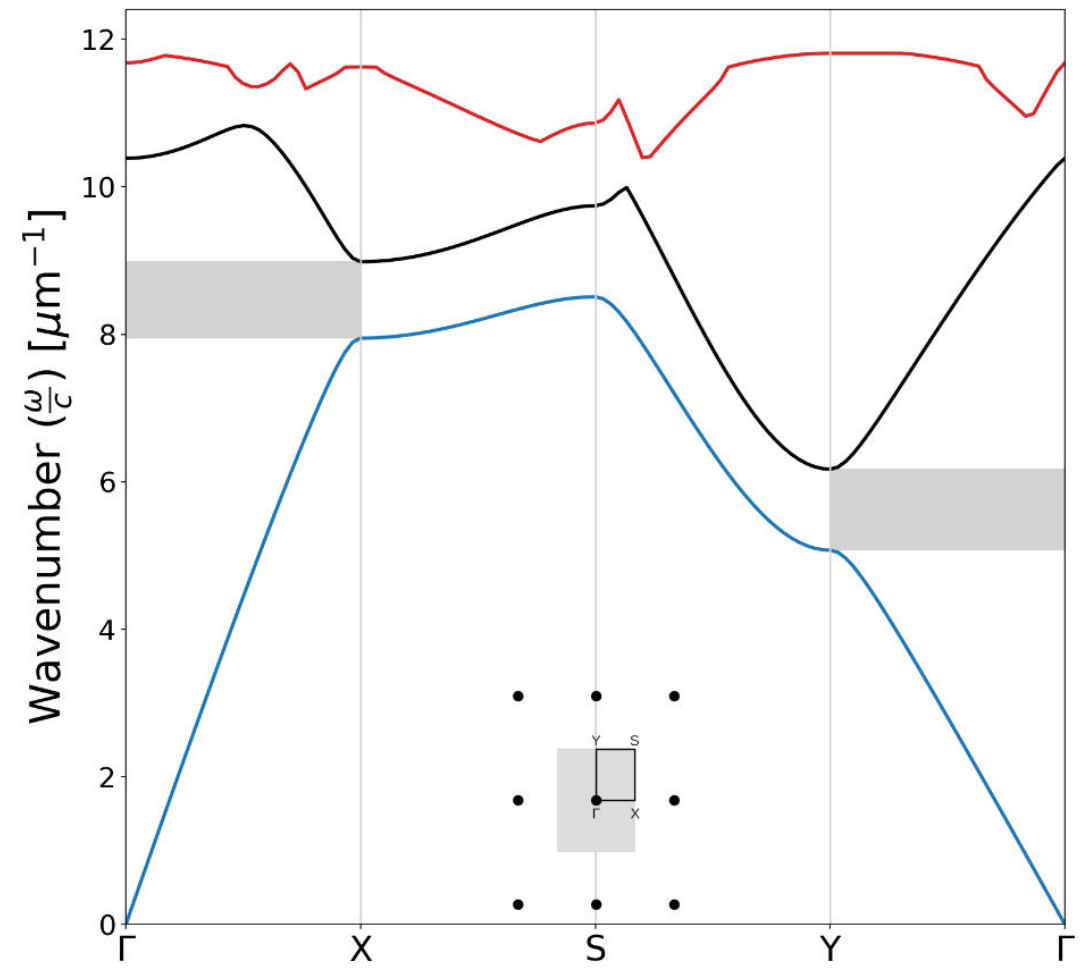

Figure 31. Band structure of a dielectric-gold PhPl crystal in a rectangular lattice with $a_{x}=300 \mathrm{~nm}, a_{y}=480 \mathrm{~nm}$ and $d=235 \mathrm{~nm}$.

Using the same parameters, the band structure of a dielectric-silver PhPl crystal was calculated, being shown in Fig. 32. In both cases, two partial bandgaps arise in the fitting range, one in $\Gamma-\mathrm{X}$ path and the other in the $\mathrm{Y}-\Gamma$ path. The former has a width of $\Delta \lambda=$ $90.95 \mathrm{~nm}$ and is centered at $\lambda_{c}=747.09 \mathrm{~nm}$, while the latter has a width of $\Delta \lambda=$ $222.11 \mathrm{~nm}$ and is centered at $\lambda_{c}=1242.28 \mathrm{~nm}$.

In addition, the first band of the band structure of both PhPl crystals is similar because the effective indexes of refraction are similar in that frequency range. The above means that the dielectric-gold and dielectric-silver $\mathrm{PhPl}$ crystals have almost the same optical response in that range of the electromagnetic spectrum.

However, the third band presents significant differences, since the inverse of the effective index of refraction has a wider fitting range. Besides, the dielectric-silver PhPl crystal can operate over a wider frequency range.

On the other hand, as shown above, for a square lattice with a low contrast of dielectric constants (in particular $\epsilon_{b} / \epsilon_{a}=2.2$ ), there were no complete bandgaps. However, using the theoretical model to analyze a rectangular lattice, and considering an 
analogous path to the square lattice, (in this case $\Gamma-\mathrm{X}-\mathrm{S}-\Gamma$ ), a complete bandgap can be found for some ratios $a_{y} / a_{x}$ and filling fractions $f$.

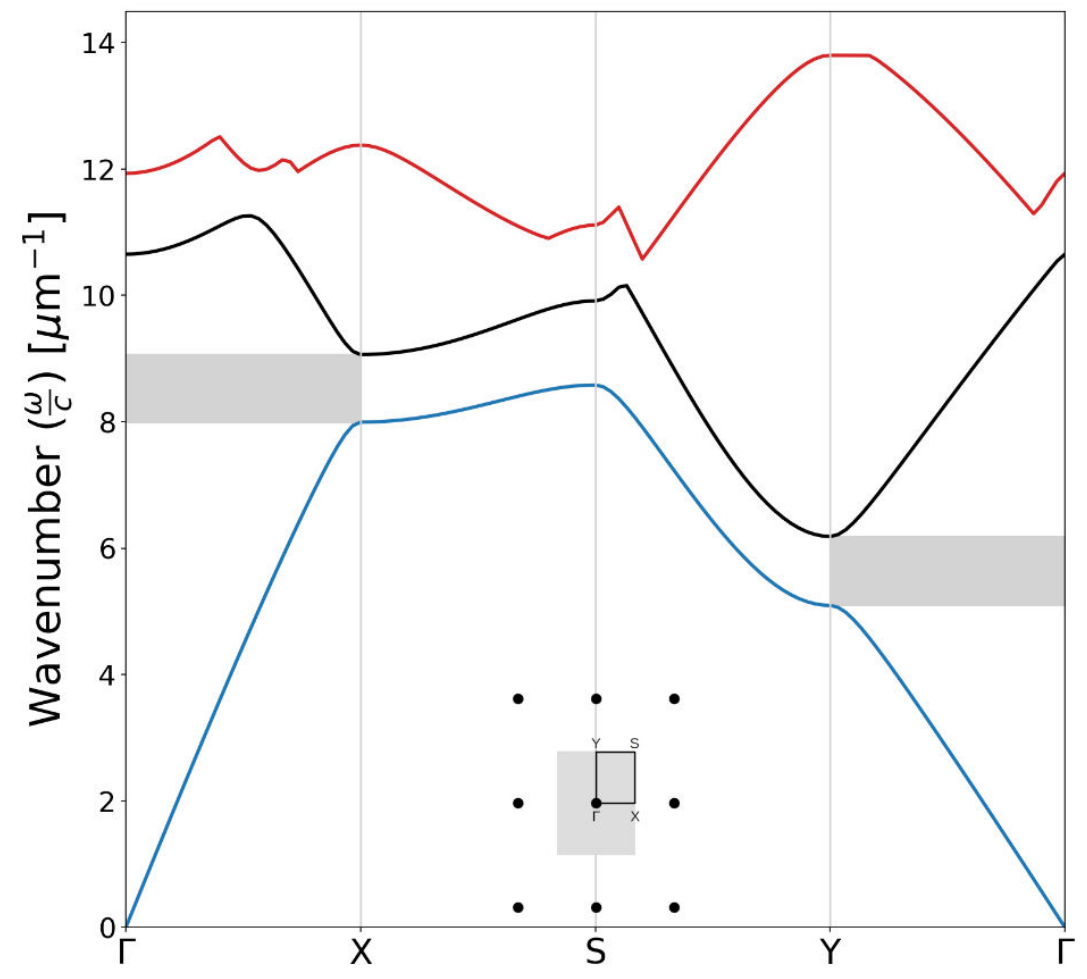

Figure 32. Band structure of a dielectric-silver PhPl crystal in a rectangular lattice with $a_{x}=300 \mathrm{~nm}, a_{y}=480 \mathrm{~nm}$ and $d=235 \mathrm{~nm}$.

Figure 33 shows the photonic band structure of a rectangular $\mathrm{PhPl}$ crystal with $a_{x}=300 \mathrm{~nm}, a_{y}=465 \mathrm{~nm}$ and $d=230 \mathrm{~nm}$. For these parameters, the bandgap center and width are $\lambda_{c}=714.12 \mathrm{~nm}$ and $\Delta \lambda=36.49 \mathrm{~nm}$, respectively. With this lattice, it is possible to have a complete bandgap even with a small index of refraction contrast considering a $\Gamma-\mathrm{X}-\mathrm{S}-\Gamma$ path.

To get a clearer picture of the bandgap formation, a heat map of the bandgap width as a function of the lattice side ratio $r_{a}=a_{y} / a_{x}$ and the filling fraction is shown in Fig. 34 . This graph corresponds to a lattice constant of $a_{x}=300 \mathrm{~nm}$, with a variation of the lattice constant $a_{y}$. This variation was between $20 \%$ to $180 \%$ of the lattice constant $a_{x}$.

In the heat map, it is possible to observe that the bandgap appears when the ratio $r_{a}=a_{y} / a_{x}$ is between 1.6 and 1.8. In addition to this, to have a complete bandgap, the filling fraction must have values between 0.1 and 0.6. In this particular case, the maximum width, $\Delta \lambda=59.79 \mathrm{~nm}$, is obtained for $r_{a}=1.5$ and $f=0.33$.

Using the forward ANN with the above parameters, $\left(a_{x}=300 \mathrm{~nm}, a_{y}=465 \mathrm{~nm}\right.$, $\left.d_{x}=d_{y}=230 \mathrm{~nm}\right)$, the predicted bandgap center and width are $\lambda_{c}=714.16 \mathrm{~nm}$ and $\Delta \lambda=30.18 \mathrm{~nm}$, respectively. For comparing, the corresponding values obtained with the 
analytical model are $\lambda_{c}=714.12 \mathrm{~nm}$ and $\Delta \lambda=36.49 \mathrm{~nm}$, respectively. For the first value, the difference is less than $1 \mathrm{~nm}$, while for the second value, the difference is

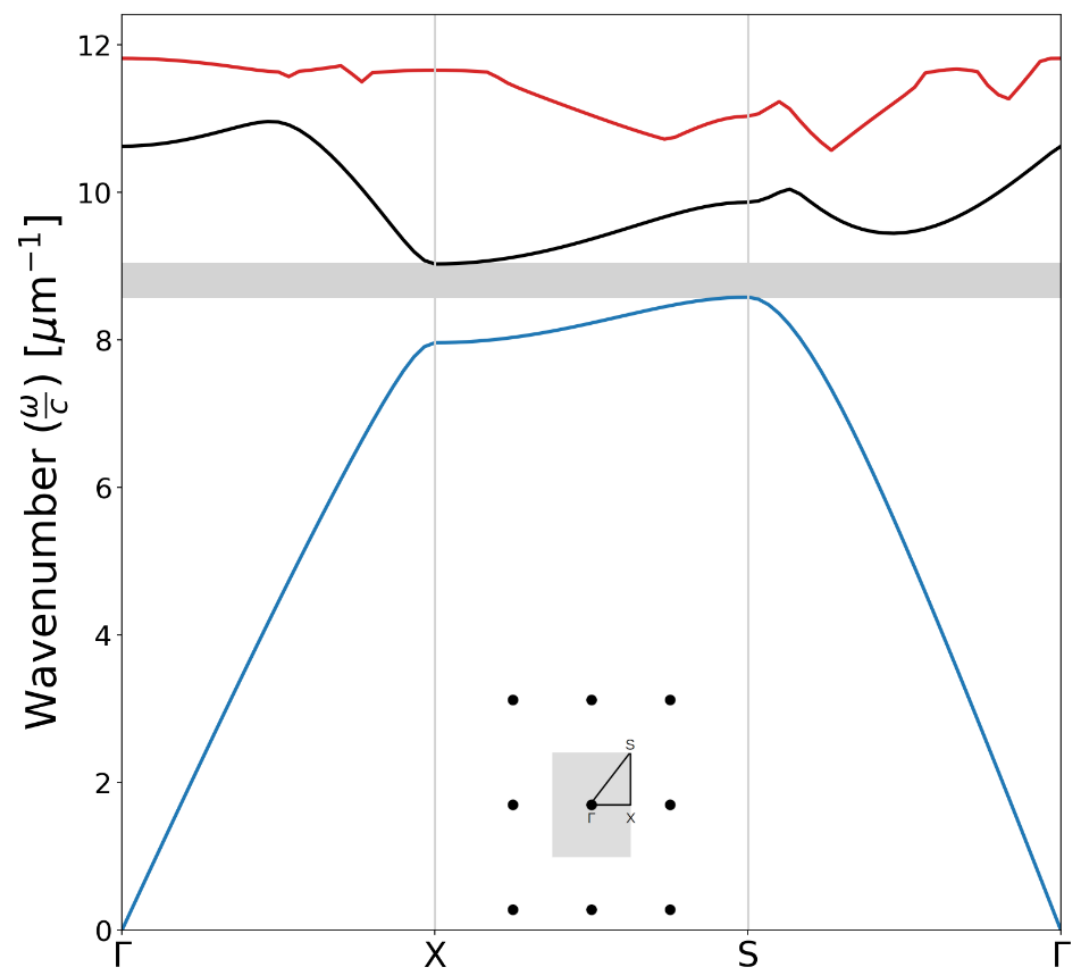

Figure 33. Band structure of a rectangular plasmonic crystal with $a_{x}=300 \mathrm{~nm}, a_{y}=465 \mathrm{~nm}$ and $d=230 \mathrm{~nm}$.

approximately of $6 \mathrm{~nm}$. 


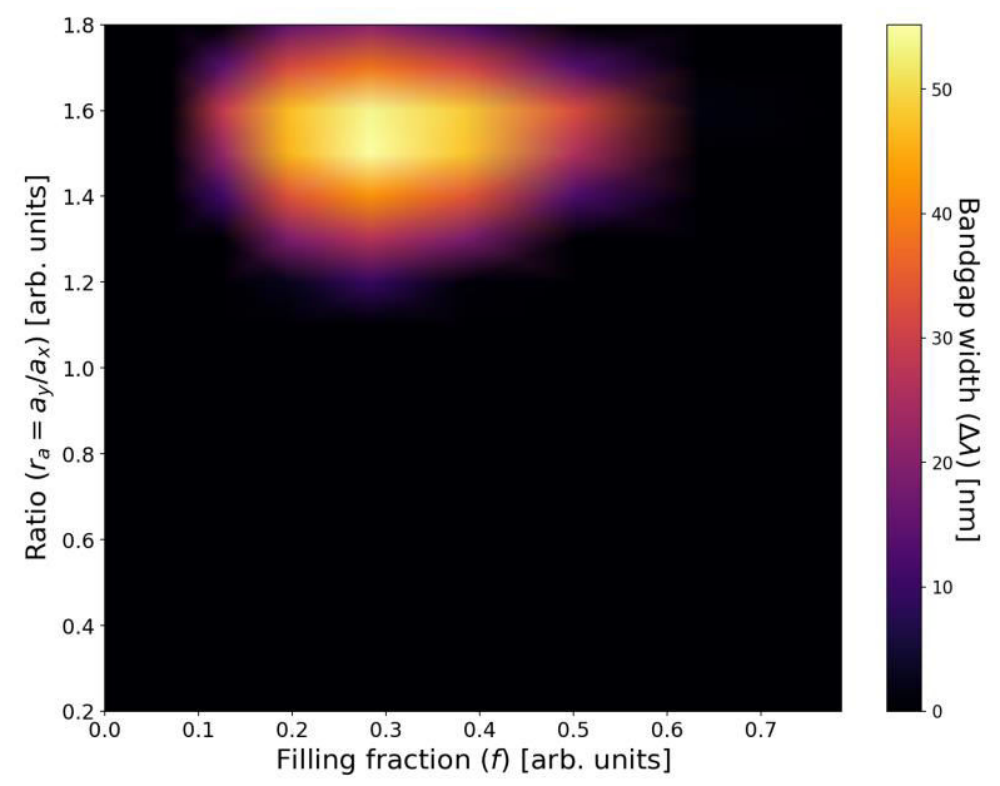

Figure 34. Bandgap width as a function of $a$ and $f$ for rectangular lattice and with an elliptical base.

Using the ANN for the inverse design, to have a bandgap centered at $\lambda_{c}=633 \mathrm{~nm}$ with a width of $\Delta \lambda=10 \mathrm{~nm}$, the algorithm predicts crystal lattice sides of $a_{x}=279.96$ $\mathrm{nm}$ and $a_{y}=339.85 \mathrm{~nm}$. For the base, it predicts axis lengths of $d_{x}=173.28 \mathrm{~nm}$ and $d_{y}=206.72 \mathrm{~nm}$. With these structural parameters, the bandgap center and width calculated with the analytical model are $\lambda_{c}=640.88 \mathrm{~nm}$ and $\Delta \lambda=10.09 \mathrm{~nm}$. The difference between the results of the algorithm and the analytical model are $7.88 \mathrm{~nm}$ for the center of gap and $0.09 \mathrm{~nm}$ for the bandgap width.

The results obtained with the analytical model are consistent with the results obtained with the ANNs. Both results are supported by numerical simulations. As for the previous case, the numerical simulations were performed for all orientations, however, for illustrative purposes, only the numerical simulations for the PhPl crystal in a particular orientation are shown; in this case in the $\Gamma-\mathrm{X}$ orientation. 


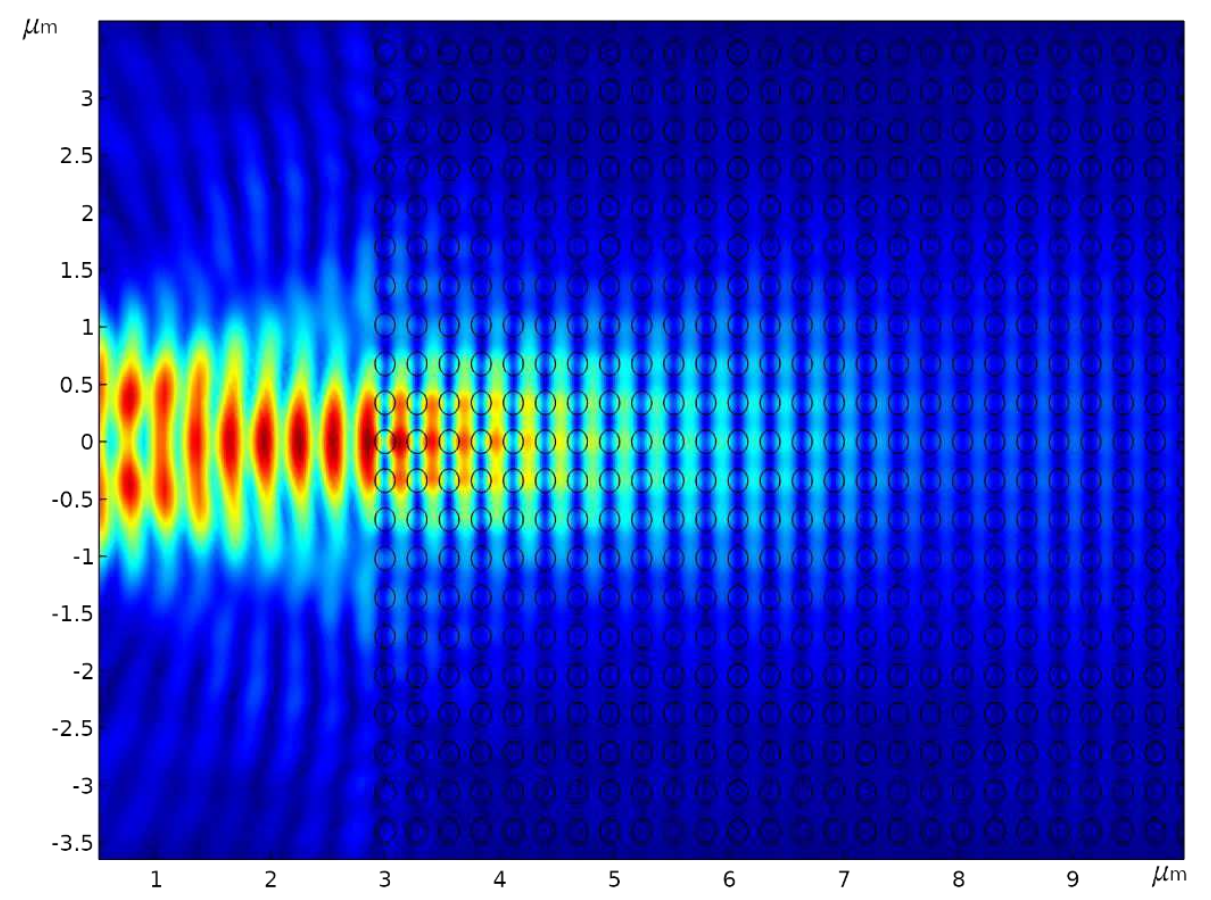

Figure 35. Numerical simulation of incident electric field at $\lambda=620 \mathrm{~nm}$ in a rectangular PhPl crystal, at an allowed frequency.

Figure 35 shows the electric field magnitud of SPPs incident at $\lambda=620 \mathrm{~nm}$, that is, at an allowed frequency.

On the contrary, Fig. 36 shows the electric field magnitud of SPPs incident at $\lambda=$ $660 \mathrm{~nm}$, a frequency within the bandgap in the $\Gamma-\mathrm{X}$ path.

Additionally, Fig. 37 shows the electric field magnitud of SPPs incident at $\lambda=750$ $\mathrm{nm}$, that is, at an allowed frequency, in the same path.

As in the triangular lattice, the SPPs are reflected in the PhPl crystal, causing the electric field intensity to increase before penetrating into the array. In Fig. 36, the extinction of SPP electric field is evident when impinging at a frequency within the bandgap. For other wavelengths, the SPPs impinge on the PhPl crystal, propagate through it and decay exponentially, as expected. 


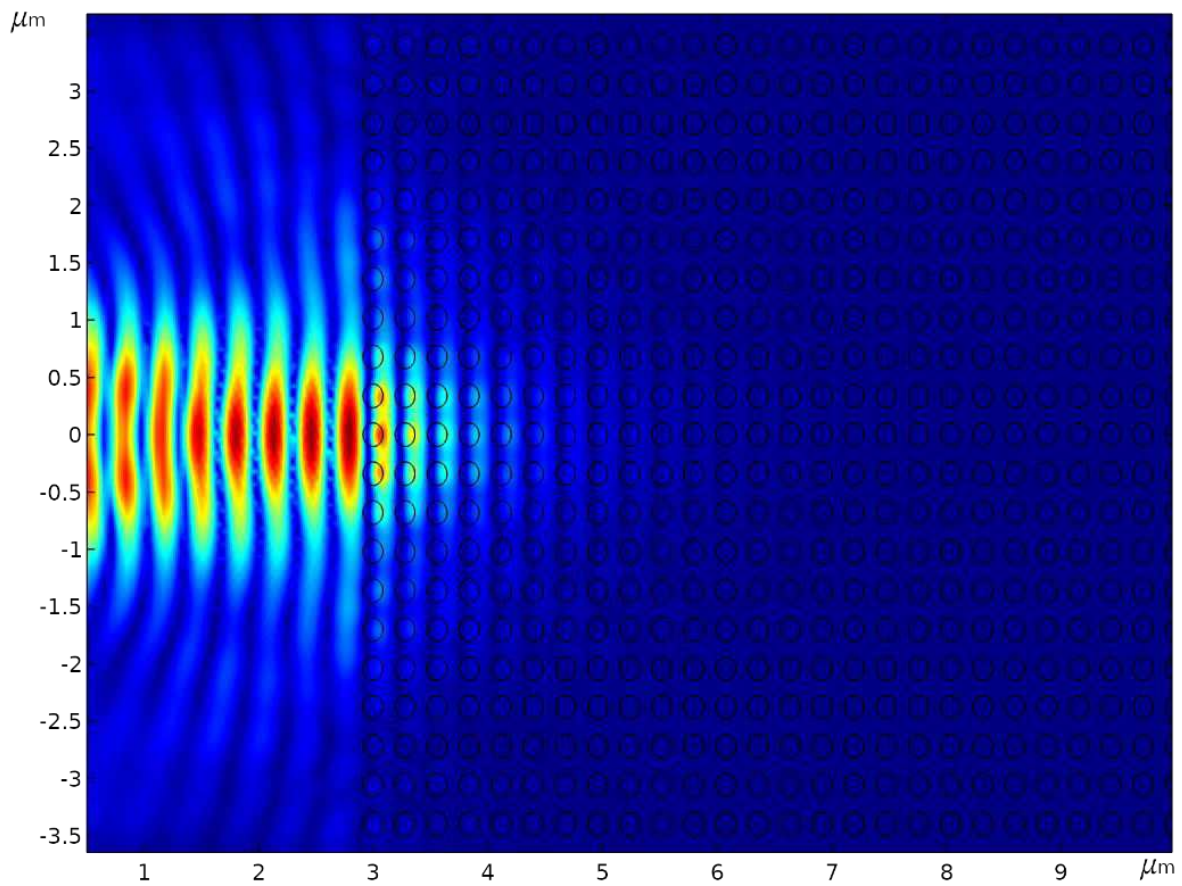

Figure 36. Numerical simulation of incident electric field at $\lambda=670$ $\mathrm{nm}$ in a rectangular $\mathrm{PhPl}$ crystal, at a frequency within the bandgap.

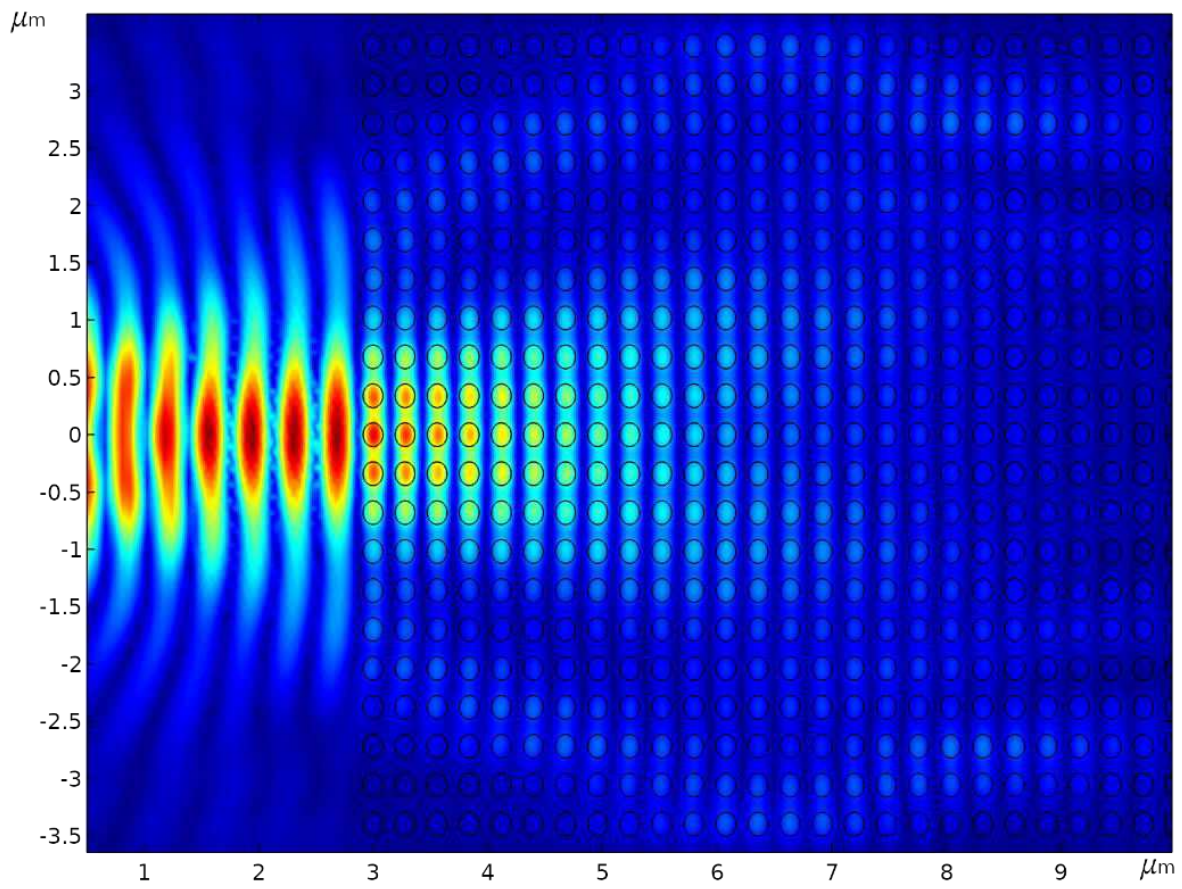

Figure 37. Numerical simulation of incident electric field at $\lambda=750$ $\mathrm{nm}$ in a rectangular PhPl crystal, at an allowed frequency.

To show clearly this fact, the normalized electric field magnitudes of SPPs for the three wavelengths are compared in Fig. 38. 


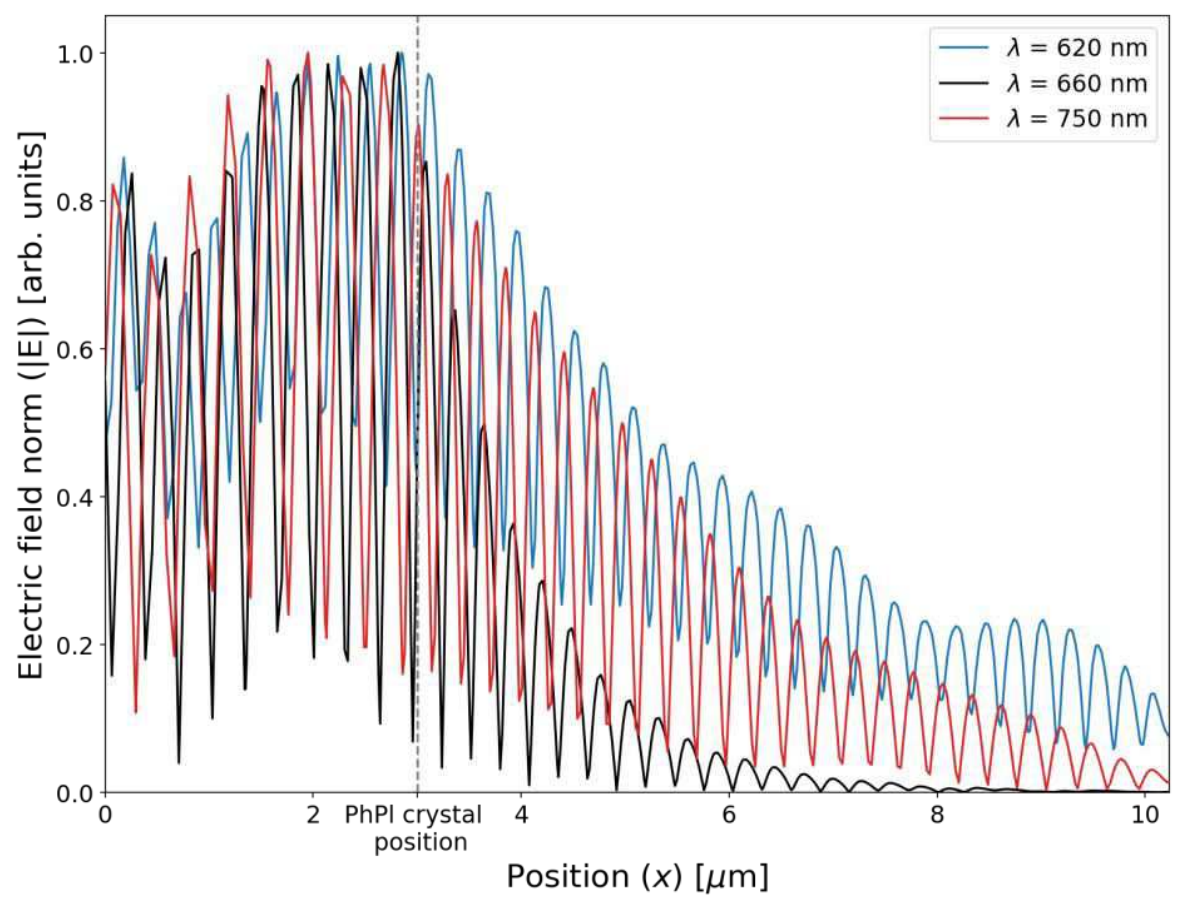

Figure 38. Comparison of the normalized absolute value of the electric field for three different wavelengths.

From the plot, it is clear that the electric field intensity for the wavelength within the bandgap decreases faster than the electric field intensities for the allowed wavelengths.

The curves in Fig. 38 reveal the field enhancement due to reflections in the PhPl crystal and the exponential decay of the electric field as it propagates into it. The above reinforces the consistent agreement between the obtained results with the analytical model and those obtained using the ANNs.

\section{Conclusions}

A general theoretical model based on PWEM is proposed to study and calculate the photonic band structure of dielectric-gold and dielectric-silver PhPl crystals. The validity and usefulness of the model are shown for several particular structures in one and two dimensions, from which, the theoretical results allow to infer that the dielectric-gold and dielectric-silver crystals respond quite similarly in the approximate range of 600-2000 nm. However, for wavelengths below $550 \mathrm{~nm}$, the dielectric-silver PhPl crystal is a better choice, since it exhibits less propagation attenuation than the dielectric-gold $\mathrm{PhPl}$ crystal.

In addition to the theoretical model, computational tools, based on ANNs, are developed to calculate the center and width of the bandgap of these hybrid PhPl systems. With this, it is possible to avoid the execution of codes that can be computationally expensive. This tool also offers the capability of tuning the optical response of the studied systems, which would imply making the design and fabrication process of these structures more efficient. 
Both, the results obtained with the analytical model and those obtained with the computational tools are consistent with the physics inherent to the systems studied, and both are supported by numerical simulations. In general, the tools proposed in this work are integrated together to study systems that could be implemented experimentally and applied in new PhPl devices.

\section{Declarations}

Funding. Jorge-Alberto Peralta-Ángeles acknowledges CONACYT support with a PhD fellowship. This work was supported by DGAPA-UNAM IN112919.

Conflict of Interests. The authors declare no competing interests.

Data Availability. The datasets generated during and/or analyzed during the current study are available from the corresponding authors on reasonable request.

Code Availability. The codes used during the current study is available from the corresponding authors on reasonable request.

Author Contribution Statement. Theoretical ideas were developed by both authors. The algorithms and simulations were developed by Jorge-Alberto Peralta-Angeles, both authors contributed to the discussion and preparation of the manuscript.

Ethics approval. Not applicable.

Consent to participate. Not applicable.

Consent for publication. Both authors consent to publication.

\section{References}

[1] Sukhoivanov, I.A., Guryev, I.V.: Photonic Crystals: Physics and Practical Modeling. Springer Series in Optical Sciences. Springer, Heidelberg, Dordrecht, London, New York (2009)

[2] Sakoda, K.: Optical Properties of Photonic Crystals. Springer, Berlin, Heidelberg, New York (2005)

[3] Joannopoulos, J.D., Johnson, S.G., Winn, J.N., Meade, R.D.: Photonic Crystals: Molding the Flow of Light. Princeton University Press, Princeton, New Jersey (2008)

[4] Maier, S.A.: Plasmonics: Fundamentals and Applications. Springer, New York (2007)

[5] Kitson, S.C., Barnes, W.L., Sambles, J.R.: Full photonic band gap for surface modes in the visible. Phys. Rev. Lett. 77, 2670-2673 (1996). https://doi.org/10.1103/PhysRevLett.77.2670

[6] Bozhevolnyi, S.I., Erland, J., Leosson, K., Skovgaard, P.M.W., Hvam, J.M.: Waveguiding in surface plasmon polariton band gap structures. Phys. Rev. Lett. 86, 30083011 (2001). https://doi.org/10.1103/PhysRevLett.86.3008 
[7] Bozhevolnyi, S.I., Volkov, V.S., Leosson, K., Boltasseva, A.: Bend loss in surface plasmon polariton band-gap structures. Applied Physics Letters 79(8), 1076-1078 (2001). https://doi.org/10.1063/1.1389507

[8] Bozhevolnyi, S.I., Volkov, V.S.: Multiple-scattering dipole approach to modeling of surface plasmon polariton band gap structures. Optics Communications 198(4), 241-245 (2001). https://doi.org/10.1016/S0030-4018(01)01516-4

[9] Drezet, A., Koller, D., Hohenau, A., Leitner, A., Aussenegg, F.R., Krenn, J.R.: Plasmonic crystal demultiplexer and multiports. Nano Letters 7(6), 1697-1700 (2007) https://arxiv.org/abs/https://doi.org/10.1021/nl070682p. https://doi.org/10.1021/n1070682p. PMID: 17500579

[10] Volkov, V.S., Bozhevolnyi, S.I., Leosson, K., Boltasseva, A.: Experimental studies of surface plasmon polariton band gap effect. Journal of Microscopy 210(3), 324-329 (2003). https://doi.org/10.1046/j.1365-2818.2003.01165.x

[11] Baudrion, A.-L., Weeber, J.-C., Dereux, A., Lecamp, G., Lalanne, P., Bozhevolnyi, S.I.: Influence of the filling factor on the spectral properties of plasmonic crystals. Phys. Rev. B 74, 125406 (2006). https://doi.org/10.1103/PhysRevB.74.125406

[12] Randhawa, S., González, M.U., Renger, J., Enoch, S., Quidant, R.: Design and properties of dielectric surface plasmon bragg mirrors. Opt. Express 18(14), 14496-14510 (2010). https://doi.org/10.1364/OE.18.014496

[13] Liu, T.-1., Russell, K.J., Cui, S., Hu, E.L.: Two-dimensional hybrid photonic/plasmonic crystal cavities. Opt. Express 22(7), 8219-8225 (2014). https://doi.org/10.1364/OE.22.008219

[14] Joseph, S., Joseph, J.: Photonic-plasmonic hybrid 2d-pillar cavity for mode confinement with subwavelength volume. IEEE Photonics Technology Letters 31(17), 1433-1436 (2019). https://doi.org/10.1109/LPT.2019.2930090

[15] Søndergaard, T., Bozhevolnyi, S.I.: Vectorial model for multiple scattering by surface nanoparticles via surface polariton-to-polariton interactions. Phys. Rev. B 67, 165405 (2003). https://doi.org/10.1103/PhysRevB.67.165405

[16] Søndergaard, T., Bozhevolnyi, S.I.: Theoretical analysis of finite-size surface plasmon polariton band-gap structures. Phys. Rev. B 71, 125429 (2005). https://doi.org/10.1103/PhysRevB.71.125429

[17] Kretschmann, M.: Phase diagrams of surface plasmon polaritonic crystals. Phys. Rev. B 68, 125419 (2003). https://doi.org/10.1103/PhysRevB.68.125419

[18] Feng, L., Lu, M.-H., Lomakin, V., Fainman, Y.: Plasmonic photonic crystal with a complete band gap for surface plasmon polariton waves. Applied Physics Letters 93(23), $231105 \quad$ (2008) https://arxiv.org/abs/https://doi.org/10.1063/1.3043581. https://doi.org/10.1063/1.3043581

[19] Mohammed, F., Quandt, A.: A simple perturbative tool to calculate plasmonic photonic bandstructures. Optical Materials 56, 107-109 (2016). https://doi.org/10.1016/j.optmat.2015.12.037. Advanced Materials for Optics, Photonics, Renewable Energies and Their Recent Advances 
[20] Ma, W., Liu, Z., Kudyshev, Z.A., Boltasseva, A., Cai, W., Liu, Y.: Deep learning for the design of photonic structures. Nature Photonics 15(2), 77-90 (2021) https://arxiv.org/abs/https://doi.org/10.1038/s41566-020-0685-y. https://doi.org/10.1038/s41566-020-0685-y

[21] Liu, D., Tan, Y., Khoram, E., Yu, Z.: Training deep neural networks for the inverse design of nanophotonic structures. ACS Photonics 5(4), 1365-1369 (2018). https://doi.org/10.1021/acsphotonics.7b01377

[22] Johnson, P.B., Christy, R.W.: Optical constants of the noble metals. Phys. Rev. B 6, 4370-4379 (1972). https://doi.org/10.1103/PhysRevB.6.4370

[23] Peters, G., Wilkinson, J.H.: ax $=\lambda b x$ and the generalized eigenproblem. SIAM Journal on Numerical Analysis 7(4), 479-492 (1970)

[24] Ruhe, A.: Algorithms for the nonlinear eigenvalue problem. SIAM Journal on Numerical Analysis 10(4), 674-689 (1973)

[25] Zhang, X., Qiu, J., Li, X., Zhao, J., Liu, L.: Complex refractive indices measurements of polymers in visible and near-infrared bands. Appl. Opt. 59(8), 2337-2344 (2020). https://doi.org/10.1364/AO.383831 